\title{
Nitrophenyl-group-containing Heterocycles. Part I. Synthesis, Characterization, Anticancer Activity and Antioxidant Properties of Some New 5,6,7,8- tetrahydro-isoquinolines Bearing 3(4)-nitrophenyl Group
}

\section{Eman M. Sayed}

Faculty of Science

\section{Reda Hassanien}

New Valley Univ

\section{Nasser Farhan}

New Valley Univer

Hanan F. Aly

National Res Center

Khaled Mahmoud

Nation Res Center

Etify A. Bakhite ( $\boldsymbol{D}$ etafy@aun.edu.eg )

Assiut University Faculty of Science

\section{Research Article}

Keywords: Synthesis, Anicancers, Antioxidants, 5,6,7,8-Tetrahydroisoquinolines, 6,7,8,9-

Tetrahydrothieno[2,3-c]isoquinolines

Posted Date: January 13th, 2022

DOI: https://doi.org/10.21203/rs.3.rs-1068683/v2

License: (c) (i) This work is licensed under a Creative Commons Attribution 4.0 International License.

Read Full License 


\section{Abstract}

Regioselective cyclocondensation of 2,4-diacetyl-5-hydroxy-5-methyl-3-(3-nitrophenyl/4nitrophenyl)cyclohexanones $1 \mathrm{a}, \mathrm{b}$ with cyanothioacetamide afforded the corresponiing 7-acetyl-4-cyano1,6-dimethyl-6-hydroxy-8-(3-nitrophenyl/4-nitrophenyl)-5,6,7,8-tetrahydro-soquinoline-3(2H)-thiones $2 \mathbf{a}, \mathbf{b}$ in $93-96 \%$ yield. Reaction of compounds $2 \mathbf{a}, \mathbf{b}$ with some ethyl iodide, 2 -chloroacetamide (4a) or its $\mathrm{N}$-aryl derivatives 4 b-e by refluxing in ethanol, in the presence of slightly excess molar amounts of sodium acetate trihydrate, for one hour gave 3 -ethylthio-5,6,7,8-tetrahydro-isoquinoline $\mathbf{3}$ and (5,6,7,8tetrahydroisoquinolin-3-ylthio)acetamides $5 \mathrm{a}-\mathrm{i}$, respectively. On heating compounds $\mathbf{5 b - d , f , g}$ in ethanol containing a catalytic amount of sodium carbonate, they converted into their isomeric 1 -amino- $6,7,8,9-$ tetrahydrothieno[2,3-c]isoquinoline-2-carboxamides $\mathbf{6 b - d , f , g}$. Structural formulae of all synthesized compounds were characterized on the basis of their elemental analyses and spectroscopic data. Also, the biological evaluation of the synthesized isoquinolines as anticancer and antioxidant agents have been carried out and the obtained results are reported herein.

\section{Introduction}

$5,6,7,8-$ Tetrahydroisoquinoline ring system is a structural fragment of many alkaloids that are next to indole alkaloids in their abundance [1-4]. Compounds containing a 5,6,7,8-tetrahydroisoquinoline fragment are used as intermediate products in the synthesis of alkaloids [5-7], precursors to enzyme inhibitors [8,9], fungicides $[10,11]$, potassium receptor antagonists [12], and drugs for the treatment of cardiovascular diseases, bronchial asthma, tumors, and viral infections $[4,13] .5,6,7,8-$

tetrahydroisoquinoline derivatives were also shown to exhibit anticonvulsant [14-16], antibacterial [17], neurotropic [18] and antimicrobial activities [19].

On the other hand, many nitro-group-containing compounds are reported to possess versatile applications in the fields of biochemistry and medicine [20-23].

In view of the above observations, the current work was planned to synthesize and characterize of some new 5,6,7,8-tetrahyroisoquinolines and related 6,7,8,9-tetrahyro-thieno[2,3-c]isoquinolines bearinng 3nitrophenyl or 4-nitrophenyl moiety with the hope that these new compounds will find good applications in both biological and medicinal fields owing to their incorporation of various pharmacophores. Also, the applications of some synthesized compounds as anticancer and as antioxidant agents have been carried out and the obtained results are reported herein.

\section{Results And Discussions}

\section{Synthesis}

Treatment of 1,3-dicarbonyl compounds $\mathbf{1 a , b}$ with cyanothioacetamide in refluxing ethanol in the presence of piperidine as a basic catalyst resulted in regioselective cyclocondensation reaction affording 
the corresponing, 7-acetyl-8-(3-nitrophenyl or 4-nitrophenyl)-4-cyano-1,6-dimethyl-6-hydroxy-5,6,7,8tetrahydroisoquinoline-3(2H)-thiones 2 a,b in $93-96 \%$ yield (Scheme 1 ).

Reaction of compounds $\mathbf{2} \mathbf{a}, \mathbf{b}$ with some halocompounds namely; ethyl iodide, 2-chloroacetamide (4a) or $\mathrm{N}$-aryl-2-chloroacetamide (4b-e) by refluxing in ethanol, in the presence of slightly excess molar amounts of sodium acetate trihydrate, for one hour gave 3-ethylthio-5,6,7,8-tetrahydroisoquinoline 3, (5,6,7,8tetrahydroisoquinolin-3-ylthio)acetamides 5 a,e and $\mathrm{N}$-aryl-(5,6,7,8-tetrahydroisoquinolin-3ylthio)acetamides 5 b-d,fi respectively (Scheme 1 ).

On refluxing of compounds $\mathbf{5 b}-\mathbf{d}, \mathbf{f}, \mathbf{g}$ with catalytic amounts of anhydrous sodium carbonate in abs. ethanol for 2 hours, they converted into 7-acetyl-1-amino-N-aryl-5,8-dimethyl-8-hydroxy-6-(3-nitrophenyl/4nitrophenyl)-6,7,8,9-tetrahydrothieno[2,3-c] isoquinoline-2-carboxamides $\mathbf{6 b}$-d,f,g. Compounds $\mathbf{6 b - d , f , g}$ were also synthesized via heating compound $\mathbf{2} \mathbf{a}, \mathbf{b}$ with the respective $\mathrm{N}$-aryl-2-chloroacetamides $\mathbf{4 b - e}$ in abs. ethanol in the presence of slightly excess molar amounts of sodium carbonate (Scheme 1).

Cyclization of compounds $\mathbf{5 b}$-d,f,g into the corresponding 6,7,8,9-tetrahydrothieno[2,3-c]isoquinolines $\mathbf{6 b}$ $\mathbf{d}, \mathbf{f}, \mathbf{g}$ may obey intramolecular Thorpe-Ziegler cyclization which its mechanism is outlined before in our publication [24].

\section{Characterizaton}

All newly synthesized compounds were characterized on the basis of their elemental analyses spectroscopic data (cf. Experimental part). Thus, IR spectra of $\mathbf{2} \mathbf{a}, \mathbf{b}$ showed characteristic absorption bands in the regions $3482-3429 \mathrm{~cm}^{-1}$ for $(\mathrm{O}-\mathrm{H}), 3235-3230 \mathrm{~cm}^{-1}$ for $(\mathrm{NH}), 2221-2220 \mathrm{~cm}^{-1}$ for $(\mathrm{C} \equiv \mathrm{N})$, and $1710-1708 \mathrm{~cm}^{-1}$ for $\left(\mathrm{C}=0\right.$, acetyl). ${ }^{1} \mathrm{H}$ NMR spectra of $2 \mathrm{a}, \mathrm{b}$ are in agreement with those of their analogues which were reported before [25]. IR spectrum of $\mathbf{3}$ revealed the disappeance of $\mathrm{NH}$ whereas its ${ }^{1} \mathrm{H}$ NMR spectrum showed the presence of ethyl group. IR spectra of $\mathbf{5 a}, \mathbf{e}$ showed absorption bands in the regions $3481-3355 \mathrm{~cm}^{-1}$ for $\left(\mathrm{OH}\right.$ and $\left.\mathrm{NH}_{2}\right), 2222-2215 \mathrm{~cm}^{-1}$ for $(\mathrm{C} \equiv \mathrm{N}), 1709-1701 \mathrm{~cm}^{-1}$ for $(\mathrm{C}=\mathrm{O}$, acetyl) and $1662-1660 \mathrm{~cm}^{-1}$ for ( $\mathrm{C}=0$, amide). ${ }^{1} \mathrm{H}$ NMR spectra of $5 \mathrm{a}$,e showed the presence of a double doublet signal corresponding to $\mathrm{SCH}_{2}$ group at $\delta$ value around 3.85 and two singlet signals at $\delta$ value overllaped with that of aromatic protons corresponding to $\mathrm{CONH}_{2}$ group [25]. IR spectra of $\mathbf{5 b} \mathbf{b}-\mathbf{d}$,f-i showed absorption bands in the regions $3563-3456 \mathrm{~cm}^{-1}$ for $(\mathrm{OH}), 3401-3289 \mathrm{~cm}^{-1}$ for $(\mathrm{NH}), 2221-2213 \mathrm{~cm}^{-1}$ for $(\mathrm{C} \equiv N), 1705-1683 \mathrm{~cm}^{-1}$ for $\left(\mathrm{C}=0\right.$, acetyl) and $1687-1666 \mathrm{~cm}^{-1}$ for $\left(\mathrm{C}=\mathrm{O}\right.$, amide). ${ }^{1} \mathrm{H}$ NMR spectra of $5 \mathrm{~b}$ d,f-i showed the presence of a double doublet signal corresponding to $\mathrm{SCH}_{2}$ group at $\delta$ value around 4.00 and a singlet signal at $\delta$ value ranged from 10.12 to 10.57 equivalent to $\mathrm{NH}$ group. IR spectra of $\mathbf{6} \mathbf{b}-\mathbf{d}, \mathbf{f}, \mathbf{g}$ revealed the disappearance of the carbonitrile band and presence of four absorption bands in the region 3517-3314 $\mathrm{cm}^{-1}$ characteristic for $\mathrm{OH}, \mathrm{NH}_{2}$ and $\mathrm{NH}$ groups beside other two bands in the regions 1705$1698 \mathrm{~cm}^{-1}$ and $1651-1624 \mathrm{~cm}^{-1}$ corresponding to acetyl group and amidic carbonyl group, respectively. ${ }^{1} \mathrm{H}$ NMR spectra of $\mathbf{6 b - d , f , g}$ showed singlet signal at $\delta$ value ranged from to 9.33-9.56 equivalent to $\mathrm{NH}$ group and a broad singlet signal referred to the amino group at $\delta$ value ranged from 7.05 to 7.13 instead of the signal of $\mathrm{SCH}_{2}$ group which exists in the ${ }^{1} \mathrm{H}$ NMR spectra of $\mathbf{5 b - d , f , g}$. The presence of tertiary 
alcoholic group in all compounds was acertained from their ${ }^{1} \mathrm{H}$ NMR spectra which possess a singlet signal at $\delta$ value ranged from 4.84 to 5.05 equivalent to one proton of $(\mathrm{OH})$ group. ${ }^{1} \mathrm{H}$ NMR spectra of all compounds displayed characteristic signals at certain $\delta$ values which are equivalent to the protons of cyclohexene ring and in accordance with those reported before for their analogues [25]. ${ }^{13} \mathrm{C}$ NMR spectra of compounds $\mathbf{5 a , c , d , f , h}$ and $\mathbf{6 b}-\mathbf{d}, \mathbf{f}, \mathbf{g}$ displayed characteristic peaks at certain $\delta$ values which are in agreement with their structures.

\section{Cytotoxic activity}

The cytotoxic activity of compounds coded with $\mathbf{2 a}, \mathbf{3}, \mathbf{5 a}, \mathbf{5 c}, \mathbf{5 d}, \mathbf{5 g}, \mathbf{5 h}$ and $\mathbf{5 i}$ against PACA2 (Pancreatic cancer cell line) and that of compounds coded with $\mathbf{5 e}, \mathbf{5 f}, \mathbf{5 g}, \mathbf{6 b}, \mathbf{6 d}, \mathbf{6 f}, \mathbf{6 g}$ against A549 (Lung carcinoma cell line) has evaluated in vitro at different concentrations ranged from 0.78 to $100 \mu \mathrm{M}$ using the MTT assay method. In this work, doxorubicin was used as a positive control drug for comparison purposes with the drug candidates $2 \mathrm{a}, 3,5 \mathrm{a}, \mathbf{5 c}, \mathbf{5 d}, \mathbf{5 e}, \mathbf{5 f}, \mathbf{5 g}, \mathbf{5 h}, \mathbf{5 i}, \mathbf{6 b}, \mathbf{6 d}, \mathbf{6 f}$ and $\mathbf{6 g}$ under the same experimental conditions. Different concentrations of these compounds were tested to reach the concentration which could cause death for $50 \%$ of the cancer cells; $I C_{50}$ value and the $I C_{50}$ range of each compound was estimated and the relation between log concentration and the probit were plot as given in Figures 1 and 2. After the cells were exposed to the solutions of the compounds under test for an incubation time of $72 \mathrm{~h}$, cytotoxic activity was determined and expressed $\mathrm{as}_{\mathrm{IC}} \mathrm{C}_{50}$ and $\mathrm{IC}_{50}$ ranges.

The results obtained (Tables 1 and 2 ) revealed that among all tested compounds: (i) four compounds 3 , $\mathbf{5 c}, \mathbf{5}$ and $\mathbf{5 i}$ showed mild to strong cytotoxic activity against PACA2 (Pancreatic cancer cell line) with $\mathrm{IC}_{50}$ of $53.5,60.1,25.9$ and $73.4 \mu \mathrm{M}$ respetively, (ii) only three compounds $\mathbf{6 b}, \mathbf{6 d}$ and $\mathbf{6 g}$ which showed considerable cytotoxic activity against A549 (Lung carcinoma cell line) with $\mathrm{IC}_{50}$ of $34.9,57.6$ and 46.3 $\mu \mathrm{M}$ respetively, (iii) compounds $5 \mathrm{~h}$ and $\mathbf{6} \mathbf{b}$ exhibit the highest activity and (iv) rest of the tested compounds being inactive against the two cell lines under investigation.

Table 1

Cytotoxic activity of compounds $\mathbf{2 a}, \mathbf{3}, \mathbf{5 a}, \mathbf{5 c}, \mathbf{5 d}, \mathbf{5 g}, \mathbf{5 h}$ and $\mathbf{5 i}$ against PACA2 (Pancreatic cancer cell line) at concentration of $100 \mu \mathrm{M}$ and their $\mathrm{IC}_{50}$ values.

\begin{tabular}{|lllllll|}
\hline \multicolumn{4}{|c|}{$95 \%$ Confidence Limits for conc. } & \multicolumn{4}{l|}{ 95\% Confidence Limits for log (conc.) } \\
\hline $\begin{array}{l}\text { Compd. } \\
\text { No. }\end{array}$ & $\begin{array}{l}\text { Estimate } \\
\mathrm{IC}_{50}(\mu \mathrm{M})\end{array}$ & $\begin{array}{l}\text { Lower } \\
\text { bound }\end{array}$ & $\begin{array}{l}\text { Upper } \\
\text { bound }\end{array}$ & $\begin{array}{l}\text { Log conc }(\mu \mathrm{M}) \text { at } \\
\text { probability 0.5 }\end{array}$ & $\begin{array}{l}\text { Lower } \\
\text { bound }\end{array}$ & $\begin{array}{l}\text { Upper } \\
\text { bound }\end{array}$ \\
\hline $\mathbf{3}$ & 53.5 & 48.349 & 59.677 & 1.728 & 1.684 & 1.776 \\
\hline $\mathbf{5 C}$ & 60.1 & 43.310 & 96.761 & 1.779 & 1.637 & 1.986 \\
\hline $\mathbf{5 h}$ & 25.9 & 21.724 & 31.121 & 1.414 & 1.337 & 1.493 \\
\hline $\mathbf{5 i}$ & 73.4 & 62.900 & 88.630 & 1.865 & 1.799 & 1.948 \\
\hline
\end{tabular}


Table 2

Cytotoxic activity of compounds $\mathbf{5 e}, \mathbf{5 f}, \mathbf{5 g}, \mathbf{6 b}, \mathbf{6 d}, \mathbf{6 f}$ and $\mathbf{6 g}$ against A549 (Lung carcinoma cell line) at concentration of $100 \mu \mathrm{M}$ and their $\mathrm{IC}_{50}$ values.

\begin{tabular}{|lllllll|}
\hline \multicolumn{4}{|c|}{$95 \%$ Confidence Limits for conc. } & \multicolumn{4}{l|}{ 95\% Confidence Limits for log (conc.) } \\
\hline $\begin{array}{l}\text { Compd. } \\
\text { No. }\end{array}$ & $\begin{array}{l}\text { Estimate } \\
\mathrm{IC}_{50}(\mu \mathrm{M})\end{array}$ & $\begin{array}{l}\text { Lower } \\
\text { bound }\end{array}$ & $\begin{array}{l}\text { Upper } \\
\text { bound }\end{array}$ & $\begin{array}{l}\text { Log conc }(\mu \mathrm{M}) \text { at } \\
\text { probability 0.5 }\end{array}$ & $\begin{array}{l}\text { Lower } \\
\text { bound }\end{array}$ & $\begin{array}{l}\text { Upper } \\
\text { bound }\end{array}$ \\
\hline 6b & 34.9 & 30.782 & 39.855 & 1.543 & 1.488 & 1.600 \\
\hline 6d & 57.6 & 49.404 & 69.055 & 1.761 & 1.694 & 1.839 \\
\hline 6 g & 46.3 & 40.490 & 53.765 & 1.666 & 1.607 & 1.731 \\
\hline
\end{tabular}

\section{Antioxidant activity}

Fourteen compounds were evaluated for DPPH scavenging activity as a measurement of their antioxidant activity. Data are represented by Mean \pm SD of 3 replicates. DPPH scavenging activity are represented as \%. Table 3 declared variable percentage of inhibition of DPPH scavenging activity of the tested compounds in a dose-dependent relationship compared with vitamin $\mathrm{C}$ as a standard. The highiest dose of synthesized compounds that is $0.10 \mu \mathrm{g} / \mathrm{mL}$ represents the highest antioxidant activity of all compounds relative to vitamin $\mathrm{C}$. The synthesized compounds $\mathbf{2 a}, \mathbf{2} \mathbf{b}, \mathbf{5 a}$ and $\mathbf{6} \mathbf{b}$ showed the highest antioxidant activity at concentration of $0.1 \mu \mathrm{g} / \mathrm{mL}$ (dose-dependent manner) . 
Table 3

DPPH Scavenging activity of isoqunioline derivatives.*

\begin{tabular}{|c|c|c|c|c|c|c|c|}
\hline Compd. & Conc. & R1 & $\mathrm{R} 2$ & R1 & $\mathbf{R} 1$ & Mean & St.De (\%) \\
\hline No. & & & & Inhibation & Inhibation & & \\
\hline $2 a$ & 0.10 & 0.016 & 0.019 & 96.72 & 96.10 & 96.41 & $0.44^{a}$ \\
\hline $2 a$ & 0.05 & 0.264 & 0.269 & 45.82 & 44.80 & 45.31 & $0.73^{b}$ \\
\hline $2 a$ & 0.01 & 0.342 & 0.344 & 29.82 & 29.41 & 29.61 & $0.29^{c}$ \\
\hline $2 b$ & 0.10 & 0.018 & 0.017 & 96.31 & 96.51 & 96.41 & $0.15^{a}$ \\
\hline $2 b$ & 0.05 & 0.019 & 0.020 & 96.10 & 95.90 & 96.00 & $0.15^{a}$ \\
\hline $2 b$ & 0.01 & 0.028 & 0.027 & 94.25 & 94.46 & 94.36 & $0.15^{\mathrm{a}}$ \\
\hline 3 & 0.10 & 0.163 & 0.166 & 66.55 & 65.93 & 66.24 & $0.44^{d}$ \\
\hline 3 & 0.05 & 0.198 & 0.202 & 59.37 & 58.55 & 58.96 & $0.58^{\mathrm{e}}$ \\
\hline 3 & 0.01 & 0.249 & 0.253 & 48.90 & 48.08 & 48.49 & $0.58^{b}$ \\
\hline $5 a$ & 0.10 & 0.021 & 0.024 & 95.69 & 95.07 & 95.38 & $0.44^{\mathrm{a}}$ \\
\hline $5 a$ & 0.05 & 0.023 & 0.021 & 95.28 & 95.69 & 95.49 & $0.29^{a}$ \\
\hline $5 a$ & 0.01 & 0.052 & 0.055 & 89.33 & 88.71 & 89.02 & $0.44^{f}$ \\
\hline $5 c$ & 0.10 & 0.127 & 0.130 & 73.94 & 73.32 & 73.63 & $0.44^{g}$ \\
\hline $5 c$ & 0.05 & 0.210 & 0.216 & 56.91 & 55.67 & 56.29 & $0.87^{e}$ \\
\hline $5 c$ & 0.01 & 0.280 & 0.297 & 42.54 & 39.05 & 40.80 & $2.47^{b}$ \\
\hline $5 d$ & 0.10 & 0.178 & 0.175 & 63.47 & 64.09 & 63.78 & $0.44^{d}$ \\
\hline $5 d$ & 0.05 & 0.269 & 0.272 & 44.80 & 44.18 & 44.49 & $0.44^{b}$ \\
\hline $5 d$ & 0.01 & 0.280 & 0.289 & 42.54 & 40.69 & 41.62 & $1.31 b$ \\
\hline $5 e$ & 0.10 & 0.078 & 0.080 & 83.99 & 83.58 & 83.79 & $0.29^{f}$ \\
\hline $5 e$ & 0.05 & 0.170 & 0.176 & 65.11 & 63.88 & 64.50 & $0.87^{d}$ \\
\hline $5 e$ & 0.01 & 0.266 & 0.269 & 45.41 & 44.80 & 45.11 & $0.44^{b}$ \\
\hline $5 f$ & 0.10 & 0.171 & 0.175 & 64.91 & 64.09 & 64.50 & $0.58^{d}$ \\
\hline
\end{tabular}


Table 3

Continued.

\begin{tabular}{|llllllll|}
\hline $\begin{array}{l}\text { Compd. } \\
\text { No. }\end{array}$ & Conc. & R1 & R2 & R1 & R1 & Mean & St.De (\%) \\
\hline $\mathbf{5 f}$ & 0.05 & 0.200 & 0.205 & 58.96 & 57.93 & 58.44 & $0.73^{\mathrm{e}}$ \\
\hline $\mathbf{5 f}$ & 0.01 & 0.241 & 0.245 & 50.54 & 49.72 & 50.13 & $0.58^{\mathrm{b}}$ \\
\hline $\mathbf{5 g}$ & 0.10 & 0.101 & 0.106 & 79.27 & 78.25 & 78.76 & $0.73^{\mathrm{g}}$ \\
\hline $\mathbf{5 g}$ & 0.05 & 0.173 & 0.177 & 64.50 & 63.68 & 64.09 & $0.58^{\mathrm{d}}$ \\
\hline $\mathbf{5 g}$ & 0.01 & 0.288 & 0.293 & 40.90 & 39.87 & 40.39 & $0.73^{\mathrm{b}}$ \\
\hline $\mathbf{5 h}$ & 0.10 & 0.187 & 0.191 & 61.63 & 60.80 & 61.21 & $0.58^{\mathrm{d}}$ \\
\hline $\mathbf{5 h}$ & 0.05 & 0.212 & 0.215 & 56.49 & 55.88 & 56.19 & $0.44^{\mathrm{e}}$ \\
\hline $\mathbf{5 h}$ & 0.01 & 0.226 & 0.229 & 53.62 & 53.01 & 53.31 & $0.44^{\mathrm{e}}$ \\
\hline $\mathbf{6 b}$ & 0.10 & 0.037 & 0.039 & 92.41 & 92.00 & 92.20 & $0.29^{\mathrm{a}}$ \\
\hline $\mathbf{6 b}$ & 0.05 & 0.042 & 0.045 & 91.38 & 90.77 & 91.07 & $0.44^{\mathrm{a}}$ \\
\hline $\mathbf{6 b}$ & 0.01 & 0.175 & 0.179 & 64.09 & 63.27 & 63.68 & $0.58^{\mathrm{d}}$ \\
\hline $\mathbf{6 f}$ & 0.10 & 0.172 & 0.174 & 64.70 & 64.29 & 64.50 & $0.29^{\mathrm{d}}$ \\
\hline $\mathbf{6 f}$ & 0.05 & 0.257 & 0.251 & 47.26 & 48.49 & 47.88 & $0.87^{\mathrm{b}}$ \\
\hline $\mathbf{6 f}$ & 0.01 & 0.298 & 0.292 & 38.85 & 40.08 & 39.46 & $0.7^{\mathrm{b}}$ \\
\hline $\mathbf{6 g}$ & 0.10 & 0.152 & 0.155 & 68.81 & 68.19 & 68.50 & $0.44^{\mathrm{d}}$ \\
\hline $\mathbf{6 g}$ & 0.05 & 0.188 & 0.191 & 61.42 & 60.80 & 61.11 & $0.44^{\mathrm{d}}$ \\
\hline $\mathbf{6 g}$ & 0.01 & 0.233 & 0.236 & 52.19 & 51.57 & 51.88 & $0.44^{\mathrm{b}}$ \\
\hline
\end{tabular}

* Data are represented by Mean \pm SD of 3 replicats. DPPH scavenging activity represented as \%. Statistical analysis is carried out using two way ANOVA coupled with CO-state computer program where similar letters are insignificant and different letters are significant at $P \leq 0.05$. Vitamin $C$ standard, was used as positive control. DPPH scavenging activity was calculated as following: $\%$ Inhibition $=100-$ [Absorbance of the test compound/Absorbance of the control] $\times 100$

The DPPH scavenging activity of the most potent compounds $\mathbf{2 a}, \mathbf{2} \mathbf{b}, \mathbf{5 a}, \mathbf{6} \mathbf{b}$ compared with that of Vitamine $\mathrm{C}$ in a dose dependent manner is given in Table 4. The DPPH scavenging activity of the latter compounds obeys the order $\mathbf{2 b}>\mathbf{5 a}>\mathbf{6 b}>\mathbf{2 a}$ (Figure 3 ). 
Table 4

DPPH scavenging activity of the potent isoqunioline compounds compared with ascorbic acid.*

\begin{tabular}{|llll|}
\hline $\begin{array}{l}\text { Compound } \\
\text { No. }\end{array}$ & $\begin{array}{l}\text { \% of inhibition at dose } \mathbf{1 0} \\
\text { micro of } \mathbf{0 . 1 9 m}\end{array}$ & $\begin{array}{l}\text { \% of inhibition at dose } 10 \\
\text { micro of 0.05gm }\end{array}$ & $\begin{array}{l}\text { \% of inhibition at dose } 10 \\
\text { micro of 0.01 gm }\end{array}$ \\
\hline $\begin{array}{l}\text { Ascorbic } \\
\text { acid }\end{array}$ & $99.20 \pm 4.22^{\mathrm{a}}$ & $66.70 \pm 5.32^{\mathrm{d}}$ & $48.78 \pm 2.22^{\mathrm{b}}$ \\
\hline 2a & $96.41 \pm 0.44^{\mathrm{a}}$ & $45.31 \pm 0.73^{\mathrm{b}}$ & $29.31 \pm 0.29^{\mathrm{c}}$ \\
\hline 2b & $96.41 \pm 0.15^{\mathrm{a}}$ & $96.00 \pm 0.15^{\mathrm{a}}$ & $94.36 \pm 0.15^{\mathrm{a}}$ \\
\hline 5a & $95.38 \pm 0.44^{\mathrm{a}}$ & $95.49 \pm 0.29^{\mathrm{a}}$ & $89.02 \pm 0.44^{\mathrm{a}}$ \\
\hline 6b & $92.20 \pm 0.29^{\mathrm{a}} \%$ & $91.07 \pm 0.44^{\mathrm{a}} \%$ & $63.68 \pm 0.58^{\mathrm{d}} \%$ \\
\hline
\end{tabular}

* Data are represented by Mean \pm SD of 3 replicats. DPPH scavenging activity represented as \%. Statistical analysis is carried out using two way ANOVA coupled with CO-state computer program where similar letters are insignificant and different letters are significant at $P \leq 0.05$. Vitamin $C$ standard, was used as positive control. DPPH scavenging activity was calculated as following: \% Inhibition $=100-$ [Absorbance of the test compound/Absorbance of the control] $\times 100$

\section{Conclusions}

In this paper, we have successfully synthesized 7-acetyl-4-cyano-1,6-dimethyl-6-hydroxy-8-(3nitrophenyl/4-nitrophenyl)-5,6,7,8-tetrahydrosoquinoline-3(2H)-thiones $2 \mathrm{a}, \mathrm{b}$ in excellent yields via cyclocondensation reaction of 2,4-diacetyl-5-hydroxy-5-methyl-3-(3-nitrophenyl/4-

nitrophenyl)cyclohexanones $\mathbf{1} \mathbf{a}, \mathbf{b}$ with cyanothioacetamide. Compounds $\mathbf{2} \mathbf{a}, \mathbf{b}$ were used as starting materials for synthesizing two new series of isoquinoline derivatives; 3 -substituted thio-5,6,7,8tetrahydroisoquinoline-4-carbonitriles 3 and $\mathbf{5 a - i}$, and related 1-amino- $N$-aryl-6,7,8,9-tetrahydrothieno[2,3c]isoquinoline-2-carboxamides $6 \mathrm{~b}-\mathrm{d}, \mathrm{f}, \mathrm{g}$. Structural formulae of all new compounds were characterized on the basis of their elemental analyses and spectroscopic data. Some of the synthesized compounds showed good activity as anticancer agents and excellent activity as antioxidants.

\section{Experimental Section}

\section{General}

Melting points were determined on a Gallan-Kamp apparatus and are uncorrected. The IR spectra were recorded on a Shimadzu 470 IR-spectrophotometer $\left(\mathrm{KBr} ; v_{\max }\right.$ in $\left.\mathrm{cm}^{-1}\right)$. The ${ }^{1} \mathrm{H}$ and ${ }^{13} \mathrm{C}$ NMR spectra were recorded on a Varian A5 $500 \mathrm{MHz}$ spectrometer using DMSO- $d_{6}$ (except for compounds $\mathbf{3}$ and $\mathbf{5 a}$ in $\mathrm{CDCl}_{3}$ ) as a solvent and tetramethylsilane (TMS) as internal reference. Coupling constants ( $J$ values) are given in Hertz $(\mathrm{Hz})$. The purity of the obtained products is checked by TLC. 
Reaction of 2-acetylcyclohexanones 1a,b with cyanothioacetamide; Synthesis compounds 2a,b; general method.

A mixture of compound $1 \mathrm{a}, \mathrm{b}(10 \mathrm{mmol})$, cyanothioacetamide $(10 \mathrm{mmol})$ and piperidine $(0 . .8 \mathrm{~mL}, 10$ $\mathrm{mmol})$ in ethanol $(100 \mathrm{~mL})$ was refluxed for $2 \mathrm{~h}$. The yellow crystals that formed on hot were collected, washed with methanol, dried in air to give compounds $2 \mathbf{a}, \mathbf{b}$. The purity of these products is $100 \%$ and needs no any purification.

7-Acetyl-4-cyano-1,6-dimethyl-6-hydroxy-8-(3-nitrophenyl)-5,6,7,8-tetra-hydroisoquinoline-3(2 H )-thione (2a). It was synthesized by reaction of 1 a with cyanothioacetamide. Yield: $96 \%$; m. p: $279-280^{\circ} \mathrm{C}$. IR: 3429

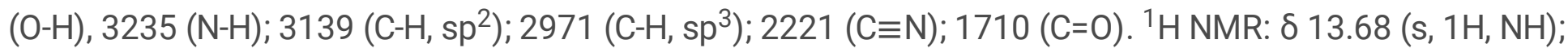
7.95-8.05 (m, 2H, ArH); 7.51-7.58 (m, 2H, ArH); $5.05(\mathrm{~s}, 1 \mathrm{H}, \mathrm{OH})$; 4.61-4.63 (d, J=10, 1H, $\left.\mathrm{C}^{8} \mathrm{H}\right)$; 3.23-3.26 (d, $\left.J=15,1 \mathrm{H}, \mathrm{C}^{5} \mathrm{H}\right), 2.88-2.90\left(\mathrm{~d}, J=10,1 \mathrm{H}, \mathrm{C}^{7} \mathrm{H}\right), 2.83-2.87\left(\mathrm{~d}, J=20,1 \mathrm{H}, \mathrm{C}^{5} \mathrm{H}\right) ; 2.12\left(\mathrm{~s}, 3 \mathrm{H}, \mathrm{COCH}_{3}\right) ; 1.86(\mathrm{~s}$, $\left.3 \mathrm{H}, \mathrm{CH}_{3}\right) ; 1.23\left(\mathrm{~s}, 3 \mathrm{H}, \mathrm{CH}_{3}\right.$ ). Anal. calcd for $\mathrm{C}_{20} \mathrm{H}_{19} \mathrm{~N}_{3} \mathrm{O}_{4} \mathrm{~S}$ (397.11): $\mathrm{C}, 60.44 ; \mathrm{H}, 4.82 ; \mathrm{N}, 10.57 \%$. Found: $\mathrm{C}$, $60.67 ; \mathrm{H}, 5.11 ; \mathrm{N}, 10.28 \%$.

7-Acetyl-4-cyano-1,6-dimethyl-6-hydroxy-8-(4-nitrophenyl)-5,6,7,8-tetra-hydroisoquinoline-3(2 H )-thione (2b). It was synthesized by reaction of $\mathbf{1 b}$ with cyanothioacetamide. Yield: $93 \%$; m. p 290-291 ${ }^{\circ} \mathrm{C}$. IR: 3482

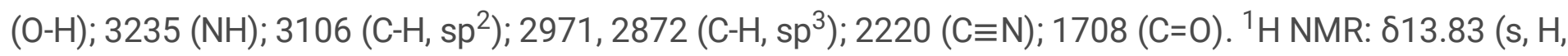
$\mathrm{NH})$, 7.84-7.86 (d, $J=10, \mathrm{H}, \mathrm{ArH}) ;$ 7.62-7.64 (d, $J=10, \mathrm{H}, \mathrm{ArH}) ;$ 7.51-7.53 (d, $J=10, \mathrm{H}, \mathrm{ArH}) ; 7.33-7.34$ (d, $J$ =5, H, Ar), $5.04(\mathrm{~s}, 1 \mathrm{H}, \mathrm{OH}) ; 4.97-4.99\left(\mathrm{~d}, J=10,1 \mathrm{H}, \mathrm{C}^{8} \mathrm{H}\right)$; 3.33-3.16 (d, $\left.J=20,1 \mathrm{H}, \mathrm{C}^{5} \mathrm{H}\right), 3.16-3.10(\mathrm{~d}, J=5$, $1 \mathrm{H}, \mathrm{C}^{7} \mathrm{H}$ ), 2.86-2.90 (d, J=20, $\left.1 \mathrm{H}, \mathrm{C}^{5} \mathrm{H}\right) ; 2.02\left(\mathrm{~s}, 3 \mathrm{H}, \mathrm{COCH}_{3}\right) ; 1.93\left(\mathrm{~s}, 3 \mathrm{H}, \mathrm{CH}_{3}\right) ; 1.29\left(\mathrm{~s}, 3 \mathrm{H}, \mathrm{CH}_{3}\right)$. Anal. calcd for $\mathrm{C}_{20} \mathrm{H}_{19} \mathrm{~N}_{3} \mathrm{O}_{4} \mathrm{~S}$ (397.11): C, 60.44; $\mathrm{H}, 4.82 ; \mathrm{N}, 10.57 \%$. Found: $\mathrm{C}, 60.32 ; \mathrm{H}, 5.04 ; \mathrm{N}, 10.33 \%$.

Reaction of compounds $2 a, b$ with ethyl iodide, 2-chloroacetamide (4a) or its N-aryl-2-chloroacetamides 4b-f; Synthesis of compounds 3 and 5a-j; general method.

A mixture of $\mathbf{2 a , b}(10 \mathrm{mmol})$, ethyl iodide, 2-chloroacetamide (4a) or $\mathrm{N}$-aryl-2-chloroacetamides $\mathbf{4 b - f}$ (10 $\mathrm{mmol})$ and sodium acetate trihydrate $(1.50 \mathrm{~g}, 11 \mathrm{mmol})$ in ethanol $(100 \mathrm{~mL})$ was refluxed for one hour. The solid that formed after cooling $(50 \mathrm{~mL})$ was collected and then recrystallized from ethanol to give white crystals of compounds $\mathbf{3}$ and $\mathbf{5 a - i}$.

7-Acetyl-4-cyano-1,6-dimethyl-3-ethylthio-6-hydroxy-8-(4-nitrophenyl)-5,6,7,8-tetrahydroisoquinoline (3): It was synthesized by reaction of $\mathbf{2 b}$ with ethyl iodide. Yield: 83\%; m.p.: 144-145 ${ }^{\circ} \mathrm{C}$. IR: 3509 (O-H); 3098 (C$\left.\mathrm{H}, \mathrm{sp}^{2}\right)$; 2974, $2919\left(\mathrm{C}-\mathrm{H}, \mathrm{sp}^{3}\right) ; 2213(\mathrm{C} \equiv \mathrm{N}) ; 1698(\mathrm{C}=\mathrm{O}), 1603(\mathrm{C}=\mathrm{N}) .{ }^{1} \mathrm{H}$ NMR: $\delta$ 8.13-8.15 (d, J=10, 2H, ArH), 7.35-7.37 (d, $J=10,2 \mathrm{H}, \mathrm{ArH}), 4.99(\mathrm{~s}, 1 \mathrm{H}, \mathrm{OH}), 4.75-4.78\left(\mathrm{~d}, J=15,1 \mathrm{H}, \mathrm{C}^{8} \mathrm{H}\right), 3.15-3.31\left(\mathrm{~m}, 3 \mathrm{H}: \mathrm{C}^{5} \mathrm{H}\right.$ and $\left.\mathrm{SCH}_{2}\right), 2.87-2.95\left(\mathrm{~m}, 2 \mathrm{H}: \mathrm{C}^{7} \mathrm{H}\right.$ and $\left.\mathrm{C}^{5} \mathrm{H}\right), 2.18\left(\mathrm{~s}, 3 \mathrm{H}, \mathrm{COCH}_{3}\right), 1.98\left(\mathrm{~s}, 3 \mathrm{H}, \mathrm{CH}_{3}\right), 1.31\left(\mathrm{~s}, 3 \mathrm{H}, \mathrm{CH}_{3}\right), 1.29$ (t, 3H, $\mathrm{CH}_{3}$ ). Anal. Calcd for $\mathrm{C}_{22} \mathrm{H}_{23} \mathrm{~N}_{3} \mathrm{O}_{4} \mathrm{~S}$ ( 425.14): C, 62.10; $\mathrm{H}, 5.45 ; \mathrm{N}, 9.88 \%$. Found: $\mathrm{C}, 62.37 ; \mathrm{H}, 5.18 ; \mathrm{N}$, $10.01 \%$. 
2-[(7-Acetyl-4-cyano-1,6-dimethyl-6-hydroxy-8-(3-nitrophenyl)-5,6,7,8-tetrahydro-isoquinolin-3-

yl)thio]acetamide (5a): It was synthesized by reaction of 2 a with 2-chloroacetamide (4a). Yield: $91 \%$; m.p.:

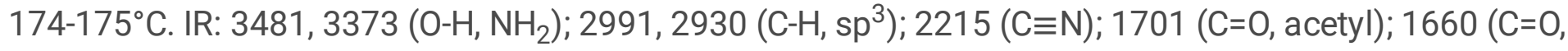
amide). ${ }^{1} \mathrm{H}$ NMR: $\delta$ 7.26-8.18 (m, $6 \mathrm{H}: \mathrm{NH}_{2}$ and $\left.\mathrm{ArH}\right), 4.53-4.55(\mathrm{~d}, J=10.0,1 \mathrm{H}, \mathrm{OH}), 3.82-3.97(\mathrm{dd}, J=$ 15.0, $2 \mathrm{H}: \mathrm{C}^{7} \mathrm{H}$ and $\left.\mathrm{C}^{8} \mathrm{H}\right), 3.02-3.21\left(\mathrm{~m}, 4 \mathrm{H}: \mathrm{SCH}_{2}\right.$ and $\left.\mathrm{C}^{5} \mathrm{H}_{2}\right), 1.96\left(\mathrm{~s}, 3 \mathrm{H}, \mathrm{COCH}_{3}\right), 1.87\left(\mathrm{~s}, 3 \mathrm{H}, \mathrm{CH}_{3}\right), 1.42(\mathrm{~s}$, $\left.3 \mathrm{H}, \mathrm{CH}_{3}\right) .{ }^{13} \mathrm{C}$ NMR: $\delta 214.79,175.43,161.92,160.77,160.44,160.11,159.78,158.14,149.88,149.66$, $145.64,134.84,131.34,129.23,123.41,122.78,118.74,116.47,114.65,114.20,111.93,106.45,69.90$, 64.12, 45.89, 42.55, 35.59, 33.60, 28.30, 25.83. Anal. Calcd. for $\mathrm{C}_{22} \mathrm{H}_{22} \mathrm{~N}_{4} \mathrm{O}_{5} \mathrm{~S}$ (454.13): C, 58.14; H, 4.88; $\mathrm{N}, 12.33 \%$. Found: $\mathrm{C}, 58.00 ; \mathrm{H}, 5.03 ; \mathrm{N}, 11.98 \%$.

2-[(7-Acetyl-4-cyano-1,6-dimethyl-6-hydroxy-8-(3-nitrophenyl)-5,6,7,8-tetrahydro-isoquinolin-3-yl)thio]- $\mathrm{N}$ phenylacetamide (5b). It was synthesized by reaction of $\mathbf{2 a}$ with $\mathrm{N}$-phenyl-2-chloroacetamide (4b).Yield:

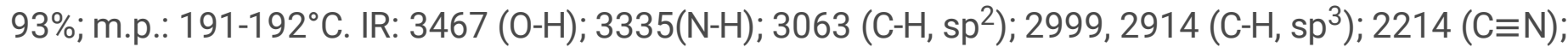
1702 (C=0, acetyl); 1687 (C=0, amide). ${ }^{1} \mathrm{H}$ NMR: $\delta 10.25(\mathrm{~s}, 1 \mathrm{H}, \mathrm{NH}), 8.06-8.08(\mathrm{~d}, 1 \mathrm{H}, \mathrm{ArH}), 7.94-7.95(\mathrm{~d}$, $1 \mathrm{H}, \mathrm{ArH}), 7.51-7.56(\mathrm{~m}, 4 \mathrm{H}, \mathrm{ArH}), 7.24-7.28(\mathrm{~m}, 2 \mathrm{H}, \mathrm{ArH}), 7.00-7.04(\mathrm{~m}, 1 \mathrm{H}, \mathrm{ArH}), 5.00(\mathrm{~s}, 1 \mathrm{H}, 0 \mathrm{H}), 4.76-4.79$ (d, $\left.J=15,1 \mathrm{H}, \mathrm{C}^{8} \mathrm{H}\right), 4.08-4.18\left(\mathrm{dd}, J=15,2 \mathrm{H}, \mathrm{SCH}_{2}\right), 3.45\left(\mathrm{~m}, 1 \mathrm{H}, \mathrm{C}^{5} \mathrm{H}\right), 2.93-2.97\left(\mathrm{~m}, 2 \mathrm{H}: \mathrm{C}^{7} \mathrm{H}\right.$ and $\left.\mathrm{C}^{5} \mathrm{H}\right)$, $2.19\left(\mathrm{~s}, 3 \mathrm{H}, \mathrm{COCH}_{3}\right), 1.91\left(\mathrm{~s}, 3 \mathrm{H}, \mathrm{CH}_{3}\right), 1.28\left(\mathrm{~s}, 3 \mathrm{H}, \mathrm{CH}_{3}\right)$. Anal. Calcd. for $\mathrm{C}_{28} \mathrm{H}_{26} \mathrm{~N}_{4} \mathrm{O}_{5} \mathrm{~S}$ (530.16): C, 63.38; $H, 4.94 ; \mathrm{N}, 10.56 \%$. Found: C, 62.99; $\mathrm{H}, 5.12 ; \mathrm{N}, 10.33 \%$.

2-[(7-Acetyl-4-cyano-1,6-dimethyl-6-hydroxy-8-(3-nitrophenyl)-5,6,7,8-tetrahydro-isoquinolin-3-yl)thio]- $\mathrm{N}$-(4tolyl)acetamide (5c). It was synthesized by reaction of $2 \mathrm{a}$ with $\mathrm{N}$-(4-tolyl)-2-chloroacetamide (4c).Yield:

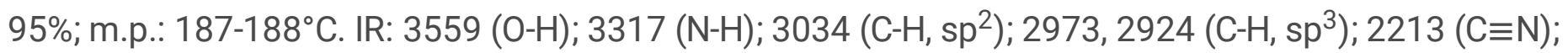
1701 (C=0, acetyl); 1675 (C=0, amide). ${ }^{1} \mathrm{H}$ NMR: $\delta 10.12$ (s, 1H, NH), 8.06-8.08 (d, 1H, ArH), 7.94-7.95 (m, $1 \mathrm{H}, \mathrm{ArH})$, 7.53-7.55 (m, 2H, Ar-H), 7.38-7.40 (d, 2H, ArH ), 7.04-7.06 (d, 2H, ArH), 4.99 (s, 1H, OH), 4.76-4.78 $\left(\mathrm{d}, J=10.0,1 \mathrm{H}, \mathrm{C}^{8} \mathrm{H}\right), 4.06-4.15\left(\mathrm{dd}, J=15,2 \mathrm{H}, \mathrm{SCH}_{2}\right), 2.89-3.32\left(\mathrm{~m}, 3 \mathrm{H}: \mathrm{C}^{7} \mathrm{H}\right.$ and $\left.\mathrm{C}^{5} \mathrm{H}_{2}\right), 2.21\left(\mathrm{~s}, 3 \mathrm{H}, \mathrm{CH}_{3}\right.$ of 4-tolyl residue), $2.17\left(\mathrm{~s}, 3 \mathrm{H}, \mathrm{COCH}_{3}\right), 1.99\left(\mathrm{~s}, 3 \mathrm{H}, \mathrm{CH}_{3}\right), 1.28\left(\mathrm{~s}, 3 \mathrm{H}, \mathrm{CH}_{3}\right) .{ }^{13} \mathrm{C}$ NMR: $\delta$ 208.74, 200.27, $181.20,165.58,160.36,157.54,150.02$, 147.75, 145.84, 136.23, 134.97, 132.03, 130.00, 128.88, 128.47, $122.54,121.56,118.87,114.90,103.87,67.23,65.74,55.86,43.11,42.28,34.55,30.84,27.33,24.51$, 20.21, 18.36. Anal. Calcd. Anal. calcd for $\mathrm{C}_{29} \mathrm{H}_{28} \mathrm{~N}_{4} \mathrm{O}_{5} \mathrm{~S}$ (544.18): C, 63.95; $\mathrm{H}, 5.18 ; \mathrm{N}, 10.29 \%$. Found: C, $64.04 ; \mathrm{H}, 4.92 ; \mathrm{N}, 9.91 \%$.

2-[(7-Acetyl-4-cyano-1,6-dimethyl-6-hydroxy-8-(3-nitrophenyl)-5,6,7,8-tetrahydro-isoquinolin-3-yl)thio]- N -(4chlorophenyl)acetamide (5d): It was synthesized by reaction of 2 a with $N$-(chlorophenyl)-2-

chloroacetamide (4d). Yield: 84\%; m.p.: 205-206 C. IR: 3536 (O-H); 3289 (N-H); 3074 (C-H, sp 2); 2973, 2924 (C-H, sp ${ }^{3}$ ); 2216 (CEN); 1694 (C=O, acetyl); 1666 (C=O, amide). ${ }^{1} \mathrm{H}$ NMR: $\delta 10.37$ (s, 1H, NH), 8.06 (d, 1H, ArH), 7.94 (s, 1H, ArH), 7.54-7.56 (m, 4H, ArH), 7.29-7.31 (d, J=10, 2H, ArH), 4.99 (s, 1H, OH), 4.76-4.78 $\left(\mathrm{d}, J=10,1 \mathrm{H} \mathrm{C}^{8} \mathrm{H}\right), 4.14-4.17\left(\mathrm{dd}, 2 \mathrm{H}, \mathrm{SCH}_{2}\right), 3.30-3.32\left(\mathrm{~d}, J=10,1 \mathrm{H}, \mathrm{C}^{5} \mathrm{H}\right), 2.93-2.95\left(\mathrm{~m}, 2 \mathrm{H}: \mathrm{C}^{7} \mathrm{H}\right.$ and $\left.\mathrm{C}^{5} \mathrm{H}\right), 2.17\left(\mathrm{~s}, 3 \mathrm{H}, \mathrm{COCH}_{3}\right), 1.89\left(\mathrm{~s}, 3 \mathrm{H}, \mathrm{CH}_{3}\right), 1.28\left(\mathrm{~s}, 3 \mathrm{H}, \mathrm{CH}_{3}\right) .{ }^{13} \mathrm{C}$ NMR: $\delta$ 204.15, 161.49, 155.77, 152.85, $145.47,143.16,141.23,133.10,130.38,125.41,123.93,123.84,122.10,117.96,116.97,115.78,110.30$, 
99.30, 62.66, 61.15, 38.53, 37.70, 30.01, 26.27, 22.75, 19.90. Anal. Calcd. for $\mathrm{C}_{28} \mathrm{H}_{25} \mathrm{ClN}_{4} \mathrm{O}_{5} \mathrm{~S}$ (564.12): C, $59.52 ; H, 4.46 ; \mathrm{N}, 9.92 \%$. Found: $C, 59.31 ; H, 4.50 ; \mathrm{N}, 10.13 \%$.

\section{2-[(7-Acetyl-4-cyano-1,6-dimethyl-6-hydroxy-8-(4-nitrophenyl)-5,6,7,8-tetrahydro-isoquinolin-3-}

yl)thio]acetamide (5e): It was synthesized by reaction of $\mathbf{2 b}$ with 2-chloroacetamide (4a).Yield: $88 \%$; m.p.:

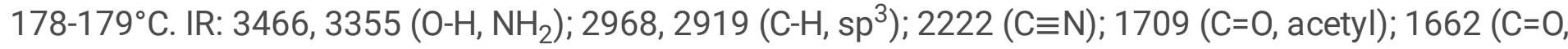
amide). ${ }^{1} \mathrm{H}$ NMR: $\delta$ 8.09-8.11 (d, $\left.J=10.0,2 \mathrm{H}, \mathrm{ArH}\right), 7.54(\mathrm{~s}, 1 \mathrm{H}, \mathrm{NH}), 7.30-7.32(\mathrm{dd}, J=5.0,2 \mathrm{H}, \mathrm{ArH}), 7.09$ (s, $1 \mathrm{H}, \mathrm{NH}), 5.00(\mathrm{~s}, 1 \mathrm{H}, \mathrm{OH}), 4.70-4.72\left(\mathrm{~d}, J=10.0,1 \mathrm{H}, \mathrm{C}^{8} \mathrm{H}\right), 3.81-3.89\left(\mathrm{dd}, J=15.0,2 \mathrm{H}, \mathrm{SCH}_{2}\right), 3.25-3.28(\mathrm{~d}$, $\left.J=15.0,1 \mathrm{H}, \mathrm{C}^{5} \mathrm{H}\right), 2.88-2.90\left(\mathrm{~d}, J=10.0,1 \mathrm{H}, \mathrm{C}^{7} \mathrm{H}\right), 2.83-2.87\left(\mathrm{~d}, J=20.0,1 \mathrm{H}, \mathrm{C}^{5} \mathrm{H}\right), 2.23\left(\mathrm{~s}, 3 \mathrm{H}, \mathrm{COCH}_{3}\right)$, $1.91\left(\mathrm{~s}, 3 \mathrm{H}, \mathrm{CH}_{3}\right), 1.24\left(\mathrm{~s}, 3 \mathrm{H}, \mathrm{CH}_{3}\right.$ ). Anal. calcd for $\mathrm{C}_{22} \mathrm{H}_{22} \mathrm{~N}_{4} \mathrm{O}_{5} \mathrm{~S}$ (454.13): C, 58.14; $\mathrm{H}, 4.88 ; \mathrm{N}, 12.33 \%$. Found: $\mathrm{C}, 57.92 ; \mathrm{H}, 4.59 ; \mathrm{N}, 12.52 \%$.

2-[(7-Acetyl-4-cyano-1,6-dimethyl-6-hydroxy-8-(4-nitrophenyl)-5,6,7,8-tetrahydro-isoquinolin-3-yl)thio]- $\mathrm{N}$ phenylacetamide (5f): It was synthesized by reaction of $\mathbf{2 b}$ with $N$-phenyl-2-chloroacetamide (4b). Yield:

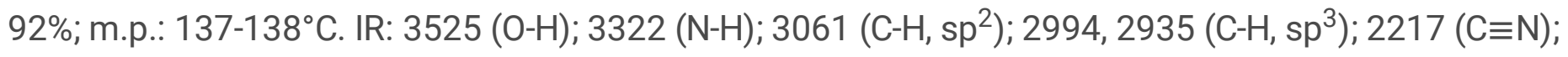
1702 (C=0, acetyl); 1687 (C=0, amide). ${ }^{1} \mathrm{H}$ NMR: $\delta 10.21$ (s, $1 \mathrm{H}, \mathrm{NH}$ ); 8.09-8.11 (d, $\left.J=10.0,2 \mathrm{H}, \mathrm{ArH}\right)$; 7.507.52 (d, $J=10,2 \mathrm{H}, \mathrm{ArH}) ; 7.32-7.33$ (d, $J=5,2 \mathrm{H}, \mathrm{ArH}) ; 7.23-7.24(\mathrm{~d}, J=5,2 \mathrm{H}, \mathrm{ArH}) ; 6.98-7.01$ (t, $J=5,1 \mathrm{H}$, ArH); $4.98(\mathrm{~s}, 1 \mathrm{H}, \mathrm{OH}) ; 4.73-4.75\left(\mathrm{~d}, J=10,1 \mathrm{H}, \mathrm{C}^{8} \mathrm{H}\right) ; 4.08-4.16\left(\mathrm{dd}, 2 \mathrm{H}, \mathrm{SCH}_{2}\right) ; 3.28-3.30\left(\mathrm{~d}, J=10,1 \mathrm{H}, \mathrm{C}^{5} \mathrm{H}\right)$, 2.89-2.94 (m, $2 \mathrm{H}: \mathrm{C}^{7} \mathrm{H}$ and $\left.\mathrm{C}^{5} \mathrm{H}\right) ; 2.16\left(\mathrm{~s}, 3 \mathrm{H}, \mathrm{COCH}_{3}\right) ; 1.89\left(\mathrm{~s}, 3 \mathrm{H}, \mathrm{CH}_{3}\right) ; 1.28\left(\mathrm{~s}, 3 \mathrm{H}, \mathrm{CH}_{3}\right) .{ }^{13} \mathrm{C}$ NMR: $\delta$ $208.52,166.01,160.51,157.73,151.77,150.03,146.07,138.89,129.52,128.67,123.78,123.26,119.05$, $115.01,104.03,78.72,67.41,65.71,56.02,42.70,34.74,31.07,27.48,24.50,18.50$. Anal. Calcd. for $\mathrm{C}_{28} \mathrm{H}_{26} \mathrm{~N}_{4} \mathrm{O}_{5} \mathrm{~S}$ (530.16): C, 63.38; $\mathrm{H}, 4.94 ; \mathrm{N}, 10.56 \%$. Found: C, 62.99; $\mathrm{H}, 5.09 ; \mathrm{N}, 10.53 \%$.

2-[(7-Acetyl-4-cyano-1,6-dimethyl-6-hydroxy-8-(4-nitrophenyl)-5,6,7,8-tetrahydro-isoquinolin-3-yl)thio]- $\mathrm{N}$-(4tolyl)acetamide $(\mathbf{5 g})$. It was synthesized by reaction of $\mathbf{2 b}$ with $\mathrm{N}$-(4-tolyl)-2-chloroacetamide (4c).Yield:

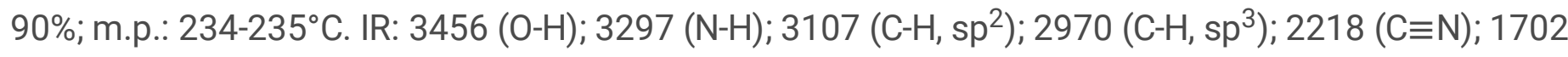
(C=0, acetyl); 1682 (C=0, amide). ${ }^{1} \mathrm{H}$ NMR: $\delta 10.12(\mathrm{~s}, 1 \mathrm{H}, \mathrm{NH}), 8.07-8.09(\mathrm{~d}, J=10,2 \mathrm{H}, \mathrm{ArH}), 7.28-7.36(\mathrm{~m}$, $4 \mathrm{H}, \mathrm{ArH}), 7.01-7.04(\mathrm{~d}, 2 \mathrm{H}, \mathrm{ArH}), 4.99(\mathrm{~s}, 1 \mathrm{H}, \mathrm{OH}), 4.68-4.71\left(\mathrm{~d}, 1 \mathrm{H}, \mathrm{C}^{8} \mathrm{H}\right), 4.05-4.07\left(\mathrm{~m}, 2 \mathrm{H}, \mathrm{SCH}_{2}\right), 3.25-$ 33.28(d, $\left.1 \mathrm{H}, \mathrm{C}^{5} \mathrm{H}\right), 2.86-2.88\left(\mathrm{~m}, 2 \mathrm{H}: \mathrm{C}^{7} \mathrm{H}\right.$ and $\left.\mathrm{C}^{5} \mathrm{H}\right), 2.18$ (s, 3H, $\mathrm{CH}_{3}$ of 4-tolyl residue), $2.13\left(\mathrm{~s}, 3 \mathrm{H}, \mathrm{COCH}_{3}\right)$, $1.84\left(\mathrm{~s}, 3 \mathrm{H}, \mathrm{CH}_{3}\right), 1.24\left(\mathrm{~s}, 3 \mathrm{H}, \mathrm{CH}_{3}\right.$ ). Anal. Calcd. for $\mathrm{C}_{29} \mathrm{H}_{28} \mathrm{~N}_{4} \mathrm{O}_{5} \mathrm{~S}$ (544.62): C, 63.95; H, 5.18; $\mathrm{N}, 10.29 \%$. Found: $\mathrm{C}, 64.08 ; \mathrm{H}, 4.91 ; \mathrm{N}, 9.93 \%$.

2-[(7-Acetyl-4-cyano-1,6-dimethyl-6-hydroxy-8-(4-nitrophenyl)-5,6,7,8-tetrahydro-isoquinolin-3-yl)thio]- N -(4chlorophenyl)acetamide $(\mathbf{5 h})$. It was synthesized by reaction of $\mathbf{2 b}$ with $\mathrm{N}$-(4-chlorophenyl)-2-

chloroacetamide (4d).Yield: 94\%; m.p.: 144-145². IR: 3563 (O-H), 3344 (N-H); 3134 (C-H, sp²); 2971, 2937 (C-H, sp $\left.{ }^{3}\right) ; 2221(\mathrm{C} \equiv \mathrm{N}) ; 1705$ (C=0, acetyl); 1681 (C=0, amide). ${ }^{1} \mathrm{H}$ NMR: $\delta 10.35$ (s, 1H, NH), 8.08-8.11 (m, $2 \mathrm{H}, \mathrm{ArH}), 7.60-7.62(\mathrm{~d}, 2 \mathrm{H}, \mathrm{ArH}), 7.29-7.54(\mathrm{~m}, 4 \mathrm{H}, \mathrm{ArH}), 4.98(\mathrm{~s}, 1 \mathrm{H}, \mathrm{OH}), 4.71-4.73(\mathrm{~d}, J=10,1 \mathrm{H}, \mathrm{CH}$ at C8), 4.06-4.14 (dd, $\left.J=15.0,2 \mathrm{H}, \mathrm{SCH}_{2}\right), 3.42-3.44\left(\mathrm{~d}, J=7.0,1 \mathrm{H}, \mathrm{C}^{5} \mathrm{H}\right), 2.90-2.92\left(\mathrm{~m}, 2 \mathrm{H}: \mathrm{C}^{7} \mathrm{H}\right.$ and $\left.\mathrm{C}^{5} \mathrm{H}\right)$, 2.15(s, 3H, $\left.\mathrm{COCH}_{3}\right), 1.85\left(\mathrm{~s}, 3 \mathrm{H}, \mathrm{CH}_{3}\right), 1.27\left(\mathrm{~s}, 3 \mathrm{H}, \mathrm{CH}_{3}\right) .{ }^{13} \mathrm{C}$ NMR: $\delta 208.53,166.23,164.75,160.47$, 
$157.63,151.75,150.04,146.07,137.85,129.52,128.73,128.60,126.83,123.77,120.90,120.53,114.98$, $103.98,67.39,65.71,55.99,43.21,42.65,34.72,31.02,27.46,24.45,18.50$. Anal. Calcd. for $\mathrm{C}_{28} \mathrm{H}_{25} \mathrm{CIN}_{4} \mathrm{O}_{5} \mathrm{~S}$ (564.12): C, 59.52; $\mathrm{H}, 4.46 ; \mathrm{N}, 9.92 \%$. Found: $\mathrm{C}, 59.20 ; \mathrm{H}, 4.67 ; \mathrm{N}, 10.07 \%$.

2-[(7-Acetyl-4-cyano-1,6-dimethyl-6-hydroxy-8-(4-nitrophenyl)-5,6,7,8-tetrahydro-isoquinolin-3-yl)thio]- N -(4acetylphenyl)acetamide (5i): It was synthesized by reaction of 2 a with $N$-(4-acetylphenyl)-2chloroacetamide (4e). Yield: 86\%; m.p.: 193-194 C. IR: 3540 (O-H); 3337(N-H); 3109 (C-H, sp $\left.{ }^{2}\right) ; 2968$ (C-H, $\left.\mathrm{sp}^{3}\right) ; 2220(\mathrm{C} \equiv \mathrm{N}) ; 1683$ (3 C=0); $1595(\mathrm{C}=\mathrm{N}) .{ }^{1} \mathrm{H}$ NMR: $\delta 10.57$ (s, 1H, NH), 8.06-8.11 (d, 2H, ArH), 7.84-7.86 (d, 2H, ArH), 7.62-7.65 (d, 2H, ArH), 7.28-7.31 (d, 2H, ArH), $5.02(\mathrm{~s}, 1 \mathrm{H}, \mathrm{OH}), 4.76-4.78\left(\mathrm{~d}, 1 \mathrm{H}, \mathrm{C}^{8} \mathrm{H}\right), 4.36-$ $4.38\left(\mathrm{~d}, 1 \mathrm{H}, \mathrm{C}^{5} \mathrm{H}\right), 4.11-4.13\left(\mathrm{dd}, 2 \mathrm{H}, \mathrm{SCH}_{2}\right), 2.88-2 . .91\left(\mathrm{~m}, 2 \mathrm{H}: \mathrm{C}^{7} \mathrm{H}\right.$ and $\left.\mathrm{C}^{5} \mathrm{H}\right), 2.12\left(\mathrm{~s}, 3 \mathrm{H}, \mathrm{COCH}_{3}\right), 1.80(\mathrm{~s}$, $\left.3 \mathrm{H}, \mathrm{COCH}_{3}\right), 1.23\left(\mathrm{~s}, 3 \mathrm{H}, \mathrm{CH}_{3}\right.$ attached to pyridine ring), $1.03\left(\mathrm{~s}, 3 \mathrm{H}, \mathrm{CH}_{3}\right)$. Anal. Calcd. for $\mathrm{C}_{30} \mathrm{H}_{28} \mathrm{~N}_{4} \mathrm{O}_{6} \mathrm{~S}$ (572.17): C, 62.92; $\mathrm{H}, 4.93 ; \mathrm{N}, 9.78 \%$. Found: $\mathrm{C}, 63.00 ; \mathrm{H}, 4.85 ; \mathrm{N}, 10.06 \%$.

\section{7-Acetyl-1-amino-2-( $\mathrm{N}$-arylcarbamoyl)-5,8-dimethyl-8-hydroxy-6-(3-nitrophenyl or 4-nitrophenyl)-6,7,8,9-} tetrahydrothieno[2,3-c ]isoquinolines $6 \mathrm{~b}-\mathrm{d}, \mathrm{f}, \mathrm{g}$; general methods.

\section{Method A)}

To a suspension of $\mathbf{5 b - d , f , g}(10 \mathrm{mmol})$ in abs. ethanol $(60 \mathrm{~mL})$, anhydrous sodium carbonate $(0.30 \mathrm{~g}))$ was added. The reaction mixture was refluxed for 3 hours. The yellow crystals that formed while hot were collected, washed with water, dried in air and then recrystallized from dioxane to give $\mathbf{6 b - d , f , g}$, repectively.

\section{7-Acetyl-1-amino-5,8-dimethyl-8-hydroxy-6-(3-nitrophenyl)- N -phenyl-6,7,8,9-tetrahydrothieno[2,3- clisoquinoline-2-carboxamide (6b).}

Yield: 87\%; m.p.: $287-288^{\circ} \mathrm{C}$. IR: 3415, 3388, $3314\left(\mathrm{O}-\mathrm{H}, \mathrm{NH}_{2}, \mathrm{~N}-\mathrm{H}\right) ; 2914$ (C-H, sp $\left.{ }^{3}\right) ; 1703$ (C=O, acetyl); 1622 (C=0, amide). ${ }^{1} \mathrm{H}$ NMR: $\delta 9.43(\mathrm{~s}, 1 \mathrm{H}, \mathrm{NH}) ; 7.31-7.84(\mathrm{~m}, 9 \mathrm{H}, \mathrm{ArH}) ; 7.09\left(\mathrm{~s}, 2 \mathrm{H}, \mathrm{NH}_{2}\right) ; 4.86-4.88$ (d, J $\left.=10,1 \mathrm{H}, \mathrm{C}^{6} \mathrm{H}\right) ; 4.84(\mathrm{~s}, 1 \mathrm{H}, \mathrm{OH}) ; 3.64-3.67\left(\mathrm{~d}, J=15,1 \mathrm{H}, \mathrm{C}^{9} \mathrm{H}\right), 3.41-3.44\left(\mathrm{~d}, J=15,1 \mathrm{H}, \mathrm{C}^{7} \mathrm{H}\right) ; 2.92-2.94(\mathrm{~d}, J$ $\left.=10,1 \mathrm{H}, \mathrm{C}^{9} \mathrm{H}\right) ; 2.21\left(\mathrm{~s}, 3 \mathrm{H}, \mathrm{COCH}_{3}\right) ; 2.03\left(\mathrm{~s}, 3 \mathrm{H}, \mathrm{CH}_{3}\right) ; 1.33\left(\mathrm{~s}, 3 \mathrm{H}, \mathrm{CH}_{3}\right) .{ }^{13} \mathrm{C}$ NMR: $\delta$ 209.44, 164.31, 158.22, $156.58,149.38,147.92,147.07,142.88,138.83,135.08,130.11,128.36,128.24,123.45,123.02,122.40$, $121.51,121.26,97.03,67.14,65.90,42.90,41.98,31.17,27.94,24.74$. Anal. Calcd. for $\mathrm{C}_{28} \mathrm{H}_{26} \mathrm{~N}_{4} \mathrm{O}_{5} \mathrm{~S}$ (530.16): C, 63.38; H, 4.94; N, 10.56\%. Found: C, 62.99; H, 5.12; N, 10.46\%.

\section{7-Acetyl-1-amino-5,8-dimethyl-8-hydroxy-6-(3-nitrophenyl)- N -(4-tolyl)-6,7,8,9-tetrahydrothieno[2,3- c}

]isoquinoline-2-carboxamide (6c). Yield: 92\%; m.p.: 291-292 ${ }^{\circ}$ C. IR: 3418, 3386, $3313\left(\mathrm{O}-\mathrm{H}, \mathrm{NH}_{2}, \mathrm{~N}-\mathrm{H}\right)$; 3075 $\left(\mathrm{C}-\mathrm{H}, \mathrm{sp}^{2}\right) ; 2914\left(\mathrm{C}-\mathrm{H}, \mathrm{sp}^{3}\right) ; 1706$ (C=0, acetyl); 1624 (C=O, amide). ${ }^{1} \mathrm{H}$ NMR: $\delta 9.35$ (s, 1H, NH); 7.06-8.08 (d, $J=10,1 \mathrm{H}, \operatorname{ArH}) ; 7.84(\mathrm{~s}, 1 \mathrm{H}, \mathrm{ArH}) ; 7.53-7.58(\mathrm{~m}, 4 \mathrm{H}, \mathrm{ArH}) ; 7.12-7.14(\mathrm{~d}, 2 \mathrm{H}, J=10, \mathrm{ArH}) ; 7.07\left(\mathrm{~s}, 2 \mathrm{H}, \mathrm{NH}_{2}\right)$; 4.86-4.88 (d, $\left.J=10,1 \mathrm{H}, \mathrm{C}^{6} \mathrm{H}\right) ; 4.84(\mathrm{~s}, 1 \mathrm{H}, \mathrm{OH}) ; 3.64-3.67\left(\mathrm{~d}, J=15,1 \mathrm{H}, \mathrm{C}^{9} \mathrm{H}\right), 3.41-3.45\left(\mathrm{~d}, J=20,1 \mathrm{H}, \mathrm{C}^{7} \mathrm{H}\right)$; 2.93-2.95 (d, $\left.J=10,1 \mathrm{H}, \mathrm{C}^{9} \mathrm{H}\right) ; 2.28\left(\mathrm{~s}, 3 \mathrm{H}, \mathrm{CH}_{3}\right.$ of 4-tolyl residue); $2.21\left(\mathrm{~s}, 3 \mathrm{H}, \mathrm{COCH}_{3}\right) ; 2.03\left(\mathrm{~s}, 3 \mathrm{H}, \mathrm{CH}_{3}\right)$; $1.33\left(\mathrm{~s}, 3 \mathrm{H}, \mathrm{CH}_{3}\right) .{ }^{13} \mathrm{C}$ NMR: $\delta 209.44,164.19,158.12,156.53,149.19,147.92,147.08,142.83,136.25$, $135.07,132.42,130.11,128.77,128.21,123.08,122.40,121.51,121.31,97.20,67.15,65.90,42.91,41.97$, 
31.18, 27.95, 24.73, 20.46. Anal. Calcd. for $\mathrm{C}_{29} \mathrm{H}_{28} \mathrm{~N}_{4} \mathrm{O}_{5} \mathrm{~S}$ (544.18): C, 63.95; $\mathrm{H}, 5.18 ; \mathrm{N}, 10.29 \%$. Found: $\mathrm{C}$, $64.14 ; \mathrm{H}, 4.92 ; \mathrm{N}, 9.93 \%$.

\section{7-Acetyl-1-amino- N -(4-chlorophenyl)-5,8-dimethyl-8-hydroxy-6-(3-nitrophenyl)-6,7,8,9-tetrahydrothieno[2,3-} c ]isoquinoline-2-carboxamide (6d). It was obtained by cyclization of compound 8c. Yield: 94\%; m.p.: 293$294^{\circ} \mathrm{C}$. IR: 3417, 3383, 3314 (O-H, NH $\left.2, \mathrm{~N}-\mathrm{H}\right)$; 3095 (C-H, sp2); 2967, 2916(C-H, sp $\left.{ }^{3}\right)$; 1706 (C=O, acetyl); 1622 (C=0, amide). ${ }^{1} \mathrm{H}$ NMR: $\delta 9.56(\mathrm{~s}, 1 \mathrm{H}, \mathrm{NH}) ; 7.36-8.08(\mathrm{~m}, 8 \mathrm{H}, \mathrm{ArH}) ; 7.13\left(\mathrm{~s}, 2 \mathrm{H}, \mathrm{NH}_{2}\right) ; 4.86-4.88(\mathrm{~d}, J$ $\left.=10,1 \mathrm{H}, \mathrm{C}^{6} \mathrm{H}\right) ; 4.85(\mathrm{~s}, 1 \mathrm{H}, \mathrm{OH}) ; 3.64-3.67\left(\mathrm{~d}, J=15,1 \mathrm{H}, \mathrm{C}^{9} \mathrm{H}\right), 3.40-3.44\left(\mathrm{~d}, J=20,1 \mathrm{H}, \mathrm{C}^{7} \mathrm{H}\right) ; 2.93-2.95(\mathrm{~d}, J$ $\left.=10,1 \mathrm{H}, \mathrm{C}^{9} \mathrm{H}\right) ; 2.21\left(\mathrm{~s}, 3 \mathrm{H}, \mathrm{COCH}_{3}\right) ; 2.04\left(\mathrm{~s}, 3 \mathrm{H}, \mathrm{CH}_{3}\right) ; 1.33\left(\mathrm{~s}, 3 \mathrm{H}, \mathrm{CH}_{3}\right) \cdot{ }^{13} \mathrm{C} \mathrm{NMR:}^{\circ} 209.42,164.35,158.33$, $156.65,149.62,147.92,147.04,142.94,135.07,130.10,128.27,128.23,126.96,122.95,122.65,122.41$, $121.51,96.81,67.14,65.88,42.8,41.99,31.17,27.94,24.74$. Anal. Calcd. for $\mathrm{C}_{28} \mathrm{H}_{25} \mathrm{ClN}_{4} \mathrm{O}_{5} \mathrm{~S}$ (564.12): C, $59.52 ; \mathrm{H}, 4.46 ; \mathrm{N}, 9.92 \%$. Found: $\mathrm{C}, 59.69 ; \mathrm{H}, 4.41 ; \mathrm{N}, 10.16 \%$.

\section{7-Acetyl-1-amino-5,8-dimethyl-8-hydroxy-6-(4-nitrophenyl)-N-phenyl-6,7,8,9-tetrahydrothieno[2,3-}

c]isoquinoline-2-carboxamide (6f). Yield: $91 \%$; m.p.: 285-286 ${ }^{\circ} \mathrm{C}$. IR: 3406, $3320\left(\mathrm{O}-\mathrm{H}, \mathrm{NH}_{2}, \mathrm{~N}-\mathrm{H}\right) ; 2921(\mathrm{C}-\mathrm{H}$, $\left.\mathrm{sp}^{3}\right) ; 1703$ (C=O, acetyl); 1622 (C=0, amide). ${ }^{1} \mathrm{H}$ NMR: $\delta 9.41$ (s, $\left.1 \mathrm{H}, \mathrm{NH}\right) ; 8.11-8.13$ (d, $\left.J=10,2 \mathrm{H}, \mathrm{ArH}\right)$; 7.677.69 (d, $J=10,2 \mathrm{H}, \mathrm{ArH})$; 7.28-7.33 (m, 5H, ArH); 7.08 (s, 2H, NH ); $4.84(\mathrm{~s}, 1 \mathrm{H}, \mathrm{OH}) ; 4.82-4.84$ (d, $J=10,1 \mathrm{H}$, $\left.\mathrm{C}^{6} \mathrm{H}\right)$; 3.59-3.63(d, $\left.J=20,1 \mathrm{H}, \mathrm{C}^{9} \mathrm{H}\right), 3.40-3.43\left(\mathrm{~d}, J=15,1 \mathrm{H}, \mathrm{C}^{7} \mathrm{H}\right) ; 2.87-2.89\left(\mathrm{~d}, J=10,1 \mathrm{H}, \mathrm{C}^{9} \mathrm{H}\right) ; 2.19$ (s, 3H, $\left.\mathrm{COCH}_{3}\right) ; 2.00\left(\mathrm{~s}, 3 \mathrm{H}, \mathrm{CH}_{3}\right) ; 1.32\left(\mathrm{~s}, 3 \mathrm{H}, \mathrm{CH}_{3}\right) .{ }^{13} \mathrm{C} \mathrm{NMR:} \delta 209.25,164.33,158.17,156.61,152.92,149.35$, $145.94,142.71,138.84,129.40,128.37,128.22,123.80,123.46,123.02,121.26,97.03,67.14,65.73$, $43.19,41.96,31.19,27.92,24.61$. Anal. Calcd. for $\mathrm{C}_{28} \mathrm{H}_{26} \mathrm{~N}_{4} \mathrm{O}_{5} \mathrm{~S}$ (530.16): C, 63.38; $\mathrm{H}, 4.94 ; \mathrm{N}, 10.56 \%$. Found: C, 62.98; $\mathrm{H}, 5.01 ; \mathrm{N}, 10.62 \%$.

\section{7-Acetyl-1-amino-5,8-dimethyl-8-hydroxy-6-(4-nitrophenyl)- N -(4-tolyl)-6,7,8,9-tetrahydrothieno[2,3- c} ]isoquinoline-2-carboxamide (6g). Yield: 92\%; m.p.:292-293ㄷ. IR: 3400, $3322\left(\mathrm{O}-\mathrm{H}, \mathrm{NH}_{2}, \mathrm{~N}-\mathrm{H}\right) ; 2919(\mathrm{C}-\mathrm{H}$, $\mathrm{sp}^{3}$ ); 1701 (C=O, acetyl); 1623 (C=0, amide). ${ }^{1} \mathrm{H}$ NMR: $\delta 9.33$ (s, 1H, NH); 8.11-8.13 (d, J=10, 2H, Ar-H); 7.55-7.57 (d, $J=10,2 \mathrm{H}, \mathrm{ArH})$; 7.27-7.29 (d, $J=10,2 \mathrm{H}, \mathrm{ArH})$; 7.11-7.13 (d, $J=10,2 \mathrm{H}, \mathrm{ArH}) ; 7.05\left(\mathrm{~s}, 2 \mathrm{H}, \mathrm{NH}_{2}\right)$; 4.84 (br s, $1 \mathrm{H}, \mathrm{OH}) ; 4.82-4.84\left(\mathrm{~d}, J=10,1 \mathrm{H}, \mathrm{C}^{6} \mathrm{H}\right) ; 3.59-3.62\left(\mathrm{~d}, J=15,1 \mathrm{H}, \mathrm{C}^{9} \mathrm{H}\right), 3.40-3.44(\mathrm{~d}, J=20,1 \mathrm{H}$, $\mathrm{C}^{7} \mathrm{H}$ ); 2.86-2.89 (d, $\left.J=15,1 \mathrm{H}, \mathrm{C}^{9} \mathrm{H}\right) ; 2.27$ (s, 3H, $\mathrm{CH}_{3}$ of 4-tolyl residue); $2.19\left(\mathrm{~s}, 3 \mathrm{H}, \mathrm{COCH}_{3}\right) ; 2.01(\mathrm{~s}, 3 \mathrm{H}$, $\mathrm{CH}_{3}$ ); 1.32 (s, 3H, $\left.\mathrm{CH}_{3}\right) .{ }^{13} \mathrm{C}$ NMR: $\delta$ 209.25, 164.19, 158.06, 156.55, 152.92,149.14, 145.76, 142.65, 136.26, 129.38, 128.77, 128.17, 123.79, 123.06, 121.30, 97.19, 67.13, 65.74, 43.18, 41.94, 31.18, 27.92, 24.59, 20.44. Anal. Calcd. for $\mathrm{C}_{29} \mathrm{H}_{28} \mathrm{~N}_{4} \mathrm{O}_{5} \mathrm{~S}$ (544.18): C, 63.95; $\mathrm{H}, 5.18 ; \mathrm{N}, 10.29 \%$. Found: $\mathrm{C}, 64.13 ; \mathrm{H}, 4.92$; N, $9.99 \%$.

\section{Method B).}

To mixture of $\mathbf{2} \mathbf{a}, \mathbf{b}(10 \mathrm{mmol})$ and respective $\mathrm{N}$-aryl-2-chloroacetamide $\mathbf{4 b}$-d $(10 \mathrm{mmol})$ in ethanol $(60 \mathrm{~mL})$, anhydrous sodium carbonate $(1.30 \mathrm{~g})$ was added. The resulting mixture was refluxed for 3 hours. The 
solid that formed while hot was collected, washed with water, dried in air and then recrystallized from dioxane to give compounds $6 \mathrm{~b}-\mathrm{d}, \mathrm{f}, \mathrm{g}$; yield: $80-86 \%$.

\section{Cytotoxic activty}

The cytotoxicity activity of the some synthesized compounds was determined according to the MTT method [26-28].

The pancreatic (PACA2) and human cancer lung (A549) cells were cultured in Dulbecco's modified Eagle's medium supplemented with $10 \%$ fetal bovine serum and $1 \%$ GlutaMAX. Then the cells were seeded into sterile 96 -well plates at a density of $10 \times 10^{3}$ cells/well and maintained at $37^{\circ} \mathrm{C}$ for $24 \mathrm{~h}$. Cancerous cells were exposed to compounds at concentrations of $0.75,1.75,3.125,6.250,12.500,25,50$, and $100 \mu \mathrm{M}$ for $72 \mathrm{~h}$. The media was removing and add $40 \mu \mathrm{l}$ MTT stock solution to each well. The resulting solutions were incubated for more than $4 \mathrm{~h}$. Subsequently, then add $120 \mu \mathrm{L}$ of $10 \%$ SDS as solubilising reagent. SPSS Software program was used to calculate the $\mathrm{IC}_{50}$ and $\mathrm{IC}_{50}$ ranges.

\section{Antioxidant activity}

DPPH has been used for measurement of free radical scavenging ability of antioxidants. Reduction of an alcoholic DPPH solution [29-31] in the presence of a hydrogen-donating antioxidant is the mainly step of this method. Hydrogen atom or electron-donation ability of the tested compounds were measured spectrophotometrically from the bleaching of the purple-colored ethanol solution of 2,2-diphenyl-1picrylhydrazyl (DPPH). In this study, antioxidant activity of the tested compounds was measured using the stable radical 2,2- diphenyl-1-picrylhydraziyl (DPPH). The free radical scavenging capacity of the tested compounds was determined using DPPh. A solution 1: prepared by dissolving DPPH $(0.002 \mathrm{gm})$ in ethanol ( $50 \mathrm{~mL}$ etnanol ). Solution 2: prepared by dissolving different weights $0.1,0.05,0.01$ grams of each sample in $1 \mathrm{~mL}$ of DMSO then take $10 \mu \mathrm{L}$ of each sample solution with $1 \mathrm{~mL}$ ethanol. Then mix $1 \mathrm{~mL}$ of solution 1 with $1 \mathrm{~mL}$ of solution 2 and the resulting mixture was vortexed thoroughly and left in the dark for about $30 \mathrm{~min}$. The absorbance of the mixture was spectrophotometrically measured at $\lambda_{\max }=$ $517 \mathrm{~nm}$ against blank $1 \mathrm{~mL}$ absolute ethanol and compared to the ascorbic acid (Vitamin C). DPPH radical scavenging activity (\% RSA) of compounds was calculated from the absorbance at the start (0) and after some reaction time $(\mathrm{T})$ according to the equation (1).

$(\%$ RSA $)=($ ABS-ATS $) / A B S \times 100(1)$

Where ABS is the absorbance of blank sample (DPPH) solution without the compound to be tested and ATS is the absorbance of tested sample.

\section{Declarations}

\section{Funding}

This work was not funded by any agency. 
Notes

The authors declare no competing financial interest.

\section{References}

1. Kisel VM, Kostyrko E O, Kovtunenko VA. Synthesis and biological properties of isoquinolines spirofused with carbocycles and heterocycles at position 4. Chem. Heterocycl. Compd., 2002; 38: 295-1318.

2. Potikha LM, Kovtunenko VA. Synthesis and properties of 3-aminodihydro-isoquinolines. Russ. Chem. Rev. 2009; 78: 513-533. http://dx.doi.org/10.1070/RC2009v078n06ABEH004041

3. Buske A, Busemann S, Muhlbacher J, Schmidt J, Porzel A, Bringmann G, Adam G. Antidesmone, a novel type isoquinoline alkaloid from Antidesma membranaceum (Euphorbiaceae). Tetrahedron, 1999; 55: 1079-1086. https://doi.org/10.1016/S0040-4020(98)01107-7

4. Dyachenko I V, Dyachenko VD. Cycloalka[c]pyridine Derivatives. Methods of Synthesis and Chemical Properties. Russ. J. Org. Chem. 2017; 53: 1769-1787.

5. Liou J-P, Cheng C.-Y. Total synthesis of ( \pm )-desoxycodeine-D: A novel route to the morphine skeleton. Tetrahedron Lett. 2000; 41: 915-918. https://doi.org/10.1016/S0040-4039(99)02188-7

6. Lipinska T. Microwave-induced solid-supported Fischer indolization, a key step in the total synthesis of the sempervirine type methoxy analogues. Tetrahedron Lett. 2004; 45: 8831-8834.

7. Vernier J-M, Holsenback H, Cosford N D P, Whitten J P, Menzaghi F, Reid R, Rao, T S, Sacaan, A I, Lloyd G K, Suto S M, Chavez-Noriega L E, Washburn M S, Urrutia A and Mc-Donald I A, Conformationaly restricted analogues of nicotine and anabasine. Bioorg. Med. Chem. Lett., 1998; 8: 2173-2178.

8. Peukert S, Schwahn U, Gusstegen S, Schreuder H and Hofmeister A. Poly(ADP-Ribose) Polymerase-1 (PARP-1) Inhibitors Based on a Tetrahydro-1(2H)-isoquinolinone Scaffold: Synthesis, Biological Evaluation and X-ray Crystal Structure. Synthesis, 2005; 1550-1554.

9. Wu, S C, Yoon, D, Chin J, van Kirk K, Seethala R, Golla R, He B, Harrity T, Kunselman L K, Morgan N N, Ponticiello R P, Taylor J R, Zebo R, Harper TW, Li W, Wang M, Zhang L, Sleczka BG, Nayeem A, Sheriff S, Camac DM, Mozin PE, Everlof JG, Li, Y-X, Ferraro CA, Kieltyka, K, Shon W, Vath M B, Zvyaga T A, Gordon DA and Robl JA. Discovery of 3-hydroxy-4-cyanoisoquinolines as novel, potent, and selective inhibitors of human 11b-hydroxydehydrogenase 1 (11b-HSD1). Bioorg. Med. Chem. Lett. 2011; 21: 6693-6698.

10. Al-Omran F, Elassar A-Z A and El-Khair A A. Synthesis of condensed heteroaromatics: novel synthesis of aminoquinolizinone derivatives as anti-HIV agents. Tetrahedron 2001; 57: 10163-10170. https://doi.org/10.1016/S0040-4020(01)01039-0

11. Hunt J C A, Briggs E, Clarke E D and Whittingham W G. Synthesis and SAR studies of novel antifungal 1,2,3-triazines. Bioorg. Med. Chem. Lett. 2007; 17: 5222-5226. https://doi.org/10.1016/j.bmcl.2007.06.076 
12. Hsin L-W, Chang L-T, Rothman RB, Dersch, CM, Fishback JA and Matsumoto RR. Synthesis and Opioid Activity of Enantiomeric N-Substituted 2,3,4,4a,5,6,7, 7a-Octahydro-1 H-benzofuro[3,2e]isoquinolines. J. Med. Chem. 2010; 53: 1392-1396. https://doi.org/10.1021/jm901503e

13. Chen L, Stefanac T, Turcotte N, Hu Z, Chen Y, Bedard J, May S and Jin H. Design and evaluation of dihydroisoquinolines as potent and orally bioavailable human cytomegalovirus inhibitors. Med. Chem. Lett. 2000; 10: 1477-1480. https://doi.org/10.1016/S0960-894X(00)00265-1

14. Sirakanyan SN, Akopyan EK, Paronikyan RG, Akopyan AG and Ovakimyan A A. Synthesis and anticonvulsant activiity of 7(8)-amiino derivatiives of condensed thieno[3,2-d]pyriimiidines. Pharm. Chem. J. 2016; 50: 296-300. https://doi.org/10.1007/s11094-016-1439-5

15. Paronikyan EG, Noravyan AS, Akopyan Sh F, Dzhagatspanyan IA, Nazaryan IM, and Paronikyan RG. Synthesis and anticonvulsant activiity of pyrano[4,3:4,5]pyrido[2,3-b]thieno[3,2-d]pyriimiidine deriivaatives and pyrimido[5,4:2,3]thieno[2,3-c]isoquinoline derivatives. Pharm. Chem. J. 2007; 41: 466-469.

16. Paronikyan, EG, Sirakanyan SN, Noravyan AS, Paronikyan RG and Dzhagatspanyan IA. Synthesis and anticonvulsant activiity of pyrazolo[3,4-b]pyrano (thiopyrano)[4,3-d]pyridine and pyrazolo[3,4c]isoquinoline derivatives. Pharm. Chem. J. 2001; 35: 8-10.

17. Paronikyan EG, Akopyan Sh F, Noravyan A S, Gaiosh G, Dashyan ShSh, Paronikyan RV, and Stepanyan GM. Synthesis and antibacterial activiity of $\mathrm{N}$ - amino-derivatiives of condensed pyridines. Pharm. Chem. J. 2013; 47: 257-260.

18. Paronikyan EG, Dashyan Sh Sh, Noravyan A S, Dzhagatspanyan IA, Paronikyan RG , Nazaryan I M and Akopyan, AG Synthesis and neurotropic activiity of amino derivatiives of cyclopenta[4,5]pyrido[3,2:4,5]thieno[3,2-d] pyrimidines and pyrimido [4,5:4,5]thieno[2,3c]isoquinolines. Pharm. Chem. J. 2016; 50:301-305. https://doi.org/10.1007/s11094-016-1440-z

19. Kamal AM , Radwan SM and Zaki RM. Synthesis and biological activity of pyrazolothienotetrahydroisoquinoline and [1,2,4]triazolo[3,4-a]thienotetrahydro-isoquinoline derivatives. Eur. J. Med. Chem. 2011;46:567-578. https://doi.org/10.1016/j.ejmech.2010.11.036

20. Ju, KS and Parales RE. Nitroaromatic compounds, from synthesis to biodegradation. Microbiol. Mol. Biol. Rev. 2010; 74: 250-272.

21. Noboru O. The Nitro Group in Organic Synthesis; Wiley VCH: Weinheim, Germany, 2001; pp 1-363.

22. Strauss, M. The nitroaromatic group in drug design.Pharmacology (for nonpharmacologists). Ind. Eng. Chem. Prod. Res. Dev. 1979; 18: 158-166.

23. Nepali K, Lee H-Y, Liou J-P. Nitro-Group-Containing Drugs. J. Med. Chem. 2019; 62: 2851-2893. https://doi.org/10.1021/acs.jmedchem.8b00147

24. El-Ossaily Y A , Al-Taifi, EA, Bakhite, EA, Marae IS and Zaki R M. Synthesis and characterization of new quinazolinylmethylsulfanylpyridines, quinazolinylthieno[2,3-b]pyridines and pyrido[3",2":4',5']thieno[3',2':4,5]pyrimido [6,1-b]quinazolines. Arkivoc 2019; part vi: 446-458. 
25. Ozols Al, Pelcher YE, Kalme ZA, Popelis YY, Turovskis IV and Duburs GY. Synthesis and chemical properties of 8-aryl-7-acyl-1-6-dimethyl-6-hydroxy-4-cyano-5,6,7,8-tetrahydro-3(2H)-isoquinolinones and isoquinolinethiones. Chem. Heterocycl. Compd. 1996; 32: 52-58.

26. Akkoç S, Kayser V, IIhan I O. Synthesis and In Vitro Anticancer Evaluation of Some Benzimidazolium Salts. J. heterocycl. Chem. 2019; 56: 2934-2944.

27. Akkoç S, Illhan IO, Gök Y, Upadhyay PJ, Kayser VJ. In vitro cytotoxic activities of new silver and PEPPSI palladium $N$-heterocyclic carbene complexes derived from benzimidazolium salts. Inorg. Chim. Acta. 2016; 449: 75-81

28. Akkoç S, Kayser V, IIlhan IO, Hibbs D E, Gök Y, Williams PA, Hawkins B and Lai FJ. New compounds based on a benzimidazole nucleus: synthesis, characterization and cytotoxic activity against breast and colon cancer cell lines. J. Organomet. Chem. 2017; 839: 98-107.

29. M. Fatiha, T. Abdelkader, Study of antioxidant activity of pyrimidinium betaines by DPPH radical scavenging method, Journal of Analytical \& Pharmaceutical Research, 2019; 8(2): 33-36.

30. Salem M S, Guirguis D B, El-Helw EAE, Ghareeb MA, Derbala, HAY. Antioxidant Activity of Heterocyclic Compounds Derived from 4-(4-Acetamidophenyl)-4-oxobut-2-enoic Acid. Intern. J. Sci. \& Res. 2014; 3: 1274-1282.

31. Wilhelm EA, Ferreira ATA, Pinz MP, dosReis AAS, Vogt AG, Stein , Zeni G and Luchese C, Antioxidant effect of quinoline derivatives containing or not selenium: Relationship with antinociceptive action quinolines are antioxidant and antinociceptive, J. Anais da Academia Brasileira de Ciências. 2017; 89:457-467.

\section{Scheme}

Scheme 1 is available in the Supplemental Files section.

\section{Figures}




\section{Probit Transformed Responses}

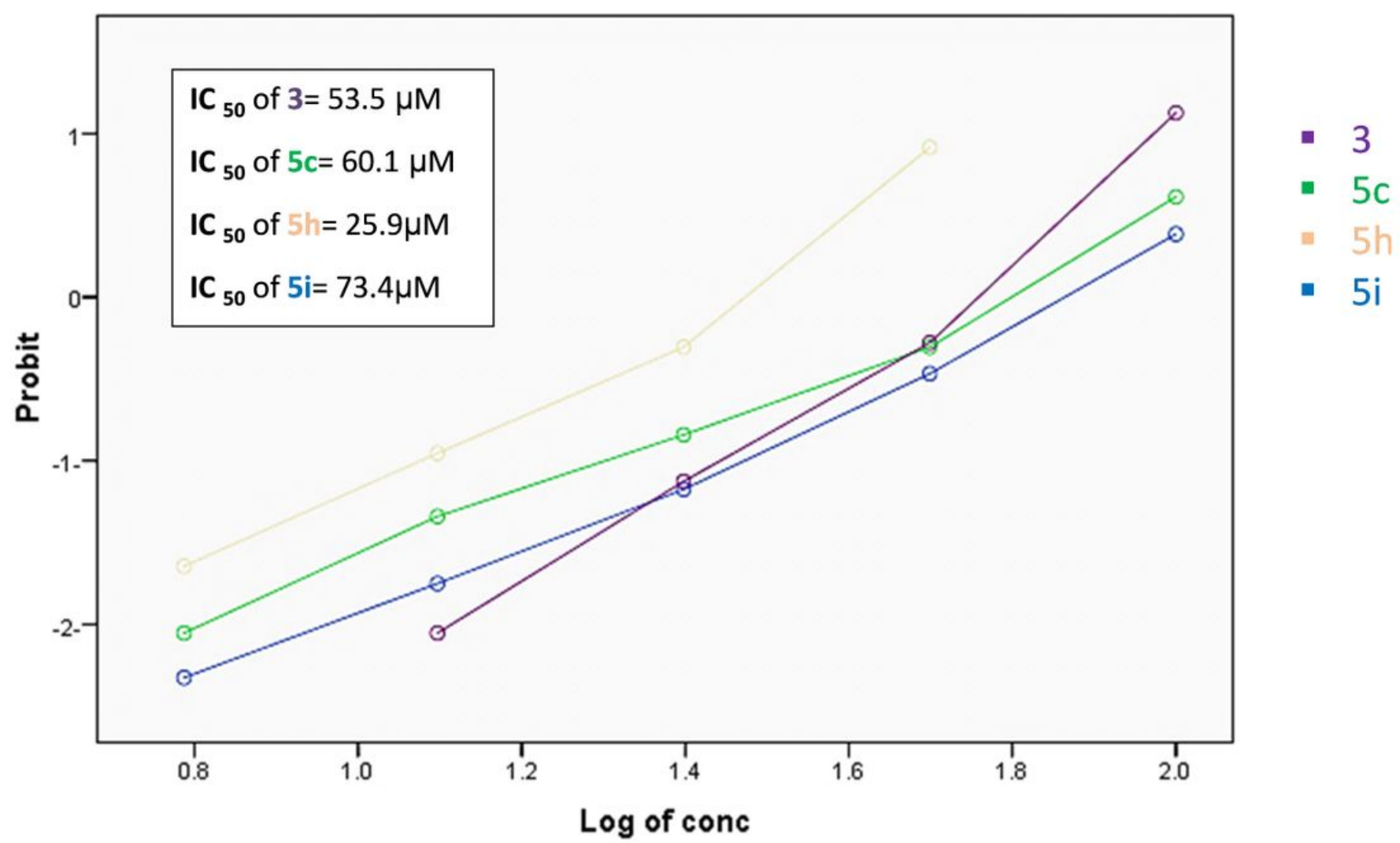

Figure 1

Cytotoxic activity of different concentrations of compounds $\mathbf{3}, \mathbf{5 c}, \mathbf{5 h}$ and $\mathbf{5 i}$ against PACA2 


\section{Probit Transformed Responses}

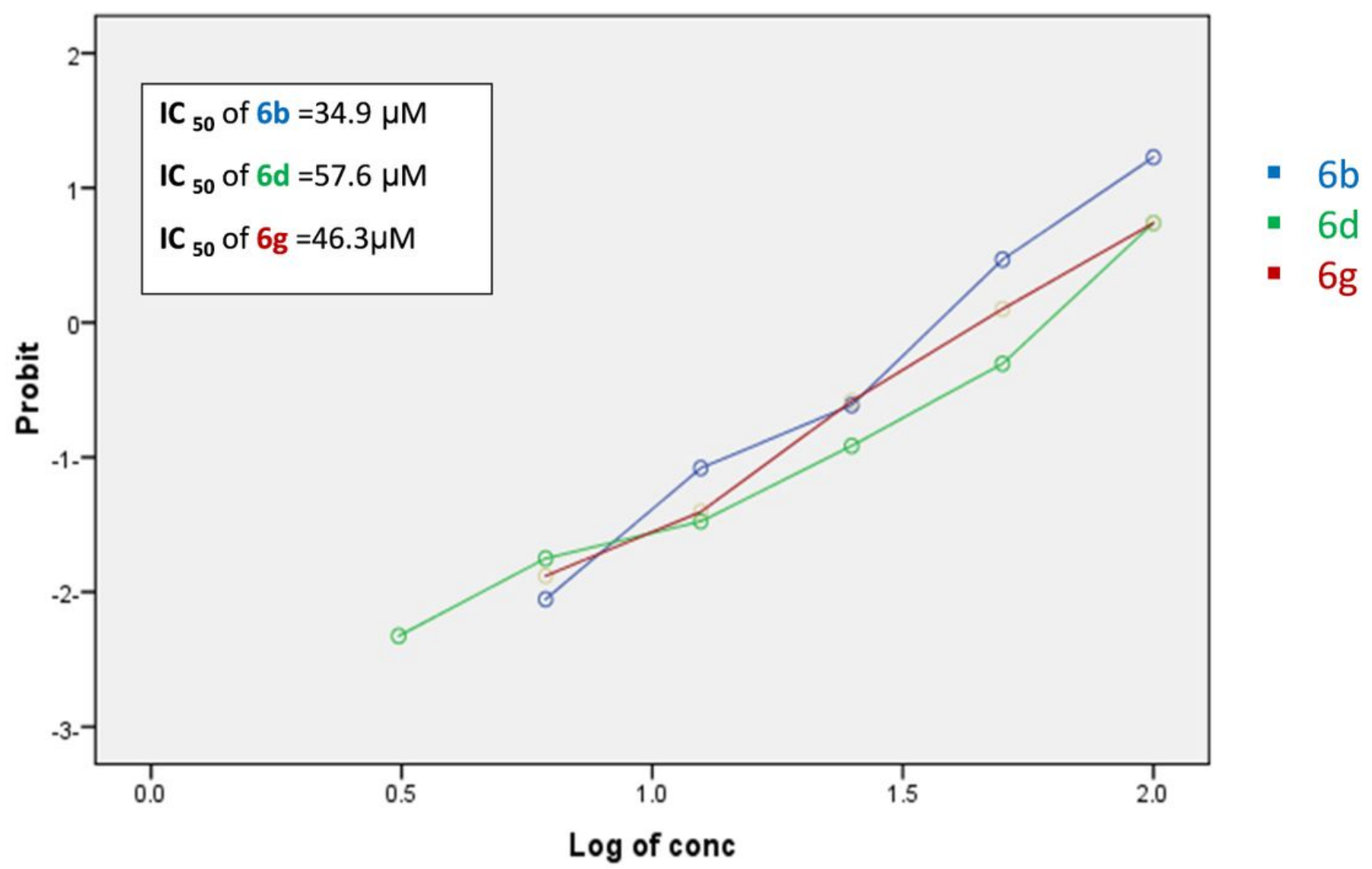

Figure 2

Cytotoxic activity of different concentrations of compounds $6 \mathrm{~b}, \mathbf{6 d}$ and $\mathbf{6 g}$ against A549. 


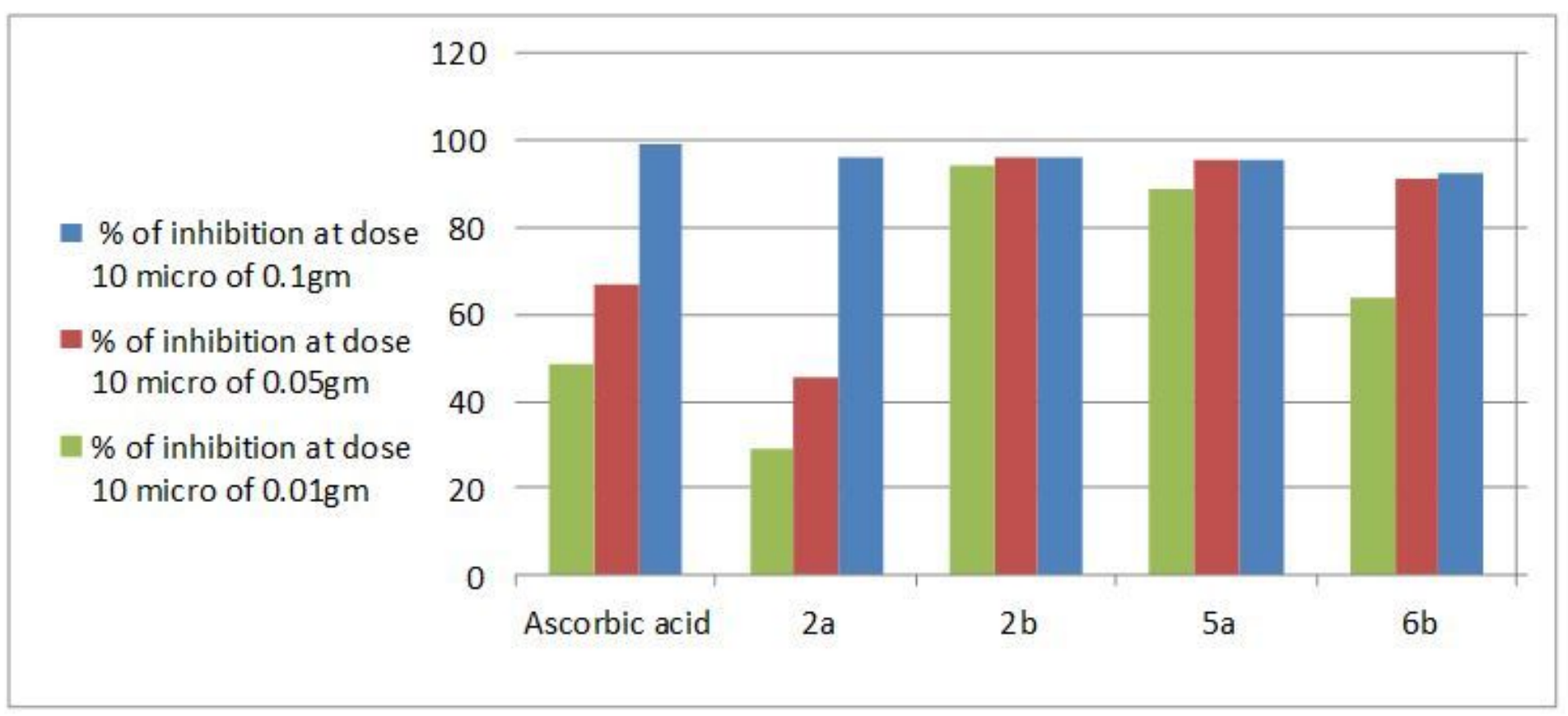

\section{Figure 3}

Antioxident activity of compounds $\mathbf{2 a}, \mathbf{2} \mathbf{b}, \mathbf{5 a}, \mathbf{6} \mathbf{b}$ and ascorbic acid as a standard.

\section{Supplementary Files}

This is a list of supplementary files associated with this preprint. Click to download.

- GraphicalAbstract.jpg

- Scheme1.jpg

- EEman22SuplementrydataforMedChemResFinal.docx 